\title{
Flora vascular de la comunidad de Bazom, Los Altos de Chiapas, MÉxico
}

\author{
Miguel Martínez-Icón', William Cetzal-Ix ${ }^{2,3,4}$, Eliana Noguera-Savelli ${ }^{1}$ \\ Y RODRIGO HÉRNANDEZ-JUÁREZ ${ }^{1}$ \\ ${ }^{1}$ El Colegio de la Frontera Sur (ECOSUR), División de Conservación de la Biodiversidad, \\ Departamento de Ecología y Sistemática Terrestre, San Cristóbal de Las Casas, Chiapas, México \\ ${ }^{2}$ El Colegio de la Frontera Sur (ECOSUR), Unidad Chetumal, Chetumal, Quintana Roo, México \\ ${ }^{3}$ Herbario $\mathrm{CICY}$, Centro de Investigación Científica de Yucatán, Mérida, Yucatán, México \\ ${ }^{4}$ Autor para la correspondencia: rolito22@hotmail.com
}

\begin{abstract}
Resumen: Se presenta un inventario florístico de la vegetación de pino-encino de la comunidad de Bazom, localizada en el municipio Huixtán, Los Altos de Chiapas, México. El listado comprende 353 taxa, repartidos en 95 familias, 235 géneros, 342 especies, tres subespecies, siete variedades y un híbrido natural. Se encontraron 55 especies bajo alguna categoría de riesgo, 52 de acuerdo a la IUCN y tres de acuerdo a la NOM-059-SEMARNAT-2010. Los hábitos más frecuentes fueron las hierbas con 225 especies, seguido por los arbustos (66), árboles (47) y trepadoras (15). El mayor número de especies fue registrado en el bosque maduro (208), seguido por el pastizal (98) y el bosque mediano (93). Los hábitos menos diversos en los diferentes tipos de vegetación fueron los árboles y las trepadoras, mientras que las hierbas presentaron la mayor diversidad. El conocimiento de la flora de Los Altos de Chiapas sigue siendo una necesidad ante la pérdida de biodiversidad debido al cambio de uso de suelo.

Palabras clave: florística, IUCN, pino-encino, NOM-059, plantas vasculares.

Abstract: A floristic inventory of oak-pine vegetation from the Bazom community located in the Huixtán municipality, in the central Highlands of Chiapas, Mexico, is presented. The list includes 353 taxa, distributed in 95 families, 235 genera, 342 species, three subspecies, seven varieties, and one natural hybrid. There are 55 species included in some category of risk, 52 according to IUCN and three according to NOM-059-SEMARNAT-2010. Herbs were the most frequent growth form with 225 species, followed by shrubs (66), and trees (47); in addition, there were 15 climbers. The greatest number of species was recorded in mature forest (208), followed by grassland (98), and medium forest (93). The less diverse growth habits were trees and climbers, while herbs presented the highest diversity. The knowledge of the flora of the Highlands of Chiapas remains necessary to face the loss of biodiversity due to changes in land use.
\end{abstract}

Key words: floristic, IUCN, NOM-059, Oak-Pine, vascular plants.

L a diversidad florística de Chiapas es considerada como una de las más importantes para México (Rzedowski, 1998), por ser el segundo estado más diverso del país (Ramírez-Marcial et al., 1998) con cerca de 10,000 especies de plantas vasculares (González-Espinosa et al., 2005). Su enorme riqueza florística es debida a su accidentada orografía y su historia geológica, que determinan una gran variedad de climas y condiciones ecológicas (Breedlove, 1981; González-Espinosa et al., 2005). De acuerdo con diferentes criterios seguidos para su identificación, en Chiapas se reconocen entre 12 y 18 formaciones vegetales de estructura y composición diferentes (González-Espinosa et al., 2005); de las cuales las asociaciones de bosque de pino, bosque de pino-encino y bosque mesófilo de montaña (Palacio-Prieto et al., 2000) ocupan cerca de $15,000 \mathrm{~km}^{2}$ de su territorio y constituyen la vegetación predominante en la Sierra Madre, Los Altos y las Montañas del Norte.

De acuerdo con el Programa Estatal de Ordenamiento Territorial (PEOT, 2005), en las últimas décadas, el cambio de uso del suelo, de forestal a agrícola-pecuario, ha tenido una velocidad alarmante en todo el estado. Cerca de la mitad de la superficie estatal se ha modificado intensamente y, aproximadamente, menos de la tercera parte de la superficie estatal permanece con vegetación primaria de sus bosques y selvas, 
distribuidas principalmente en las áreas naturales protegidas (Ramos, 2013). En Los Altos de Chiapas es evidente la conversión del bosque para agricultura, el pastoreo de ovinos o el establecimiento de nuevos centros de población. Este cambio en el uso del suelo ha provocado modificaciones en la estructura y composición florística de los remanentes forestales (Parra-Vázquez et al., 1993; González-Espinosa et al., 1997).

De acuerdo con Breedlove (1981) los estudios para el conocimiento de la flora de Chiapas se iniciaron a finales del siglo XVII. Sin embargo, aún existen zonas del estado que han sido poco muestreadas o inclusive no se han explorado (Pérez-Farrera et al., 2012). La importancia de los inventarios florísticos radica en que permiten detectar elementos relevantes de conservación, tales como especies en peligro de extinción, endémicas, de distribución restringida o presentes en un solo tipo de vegetación o raras.

En este sentido, la información publicada hasta el momento para la flora de la comunidad de Bazom se refiere a estudios sobre la sucesión secundaria de los Altos de Chiapas (González-Espinosa et al., 1991), la diversidad de especies asociadas a la presencia de pinos en el dosel (GonzálezEspinosa et al., 1995) y a la estructura y composición de especies arbóreas bajo un gradiente de "pinarización" en los Altos de Chiapas, México (Galindo-Jaimes et al., 2002). A pesar del conocimiento florístico y ecológico existente para la comunidad de Bazom, no se ha realizado un estudio florístico que incluya otros grupos además de los árboles.

La comunidad de Bazom fue fundada en 1960 por $20 \mathrm{fa}-$ milias, que por diferencias ideológicas, políticas y religiosas, decidieron emigrar de la comunidad de Oquem, municipio de Huixtán, Chiapas, a otras tierras y constituir una nueva comunidad que establecieron en la zona más alta del municipio de Huixtán, con una extensión de 167 ha, donde predomina la vegetación de bosque de pino-encino. El principal modo de vida de los primeros pobladores fue la agricultura tradicional, y posteriormente un grupo de pobladores, conformado por Nicolás Hernández Ton, Miguel Martínez Álvarez, Martín Pérez Ara, Nicolás Pérez Ton, Martín Pérez Gómez y Pedro Pérez Gómez se propusieron impulsar y trabajar por la consolidación del poblado como una comunidad reconocida ante el municipio, y con esto lograr contar con algunos de los servicios básicos y el establecimiento de una escuela; lo que incentivó a los jóvenes a formarse como técnicos y profesionistas en diversas áreas del conocimiento, además como promotores de la educación bilingüe (tzotzil, español). Posteriormente, muchos de estos jóvenes emigraron en busca de mejores oportunidades de empleo, con lo que paulatinamente se produjo el abandono de las actividades agrícolas en la comunidad, y una disminución de la población. En la actualidad, la mayoría de los pobladores son personas de la tercera edad y no hay quien
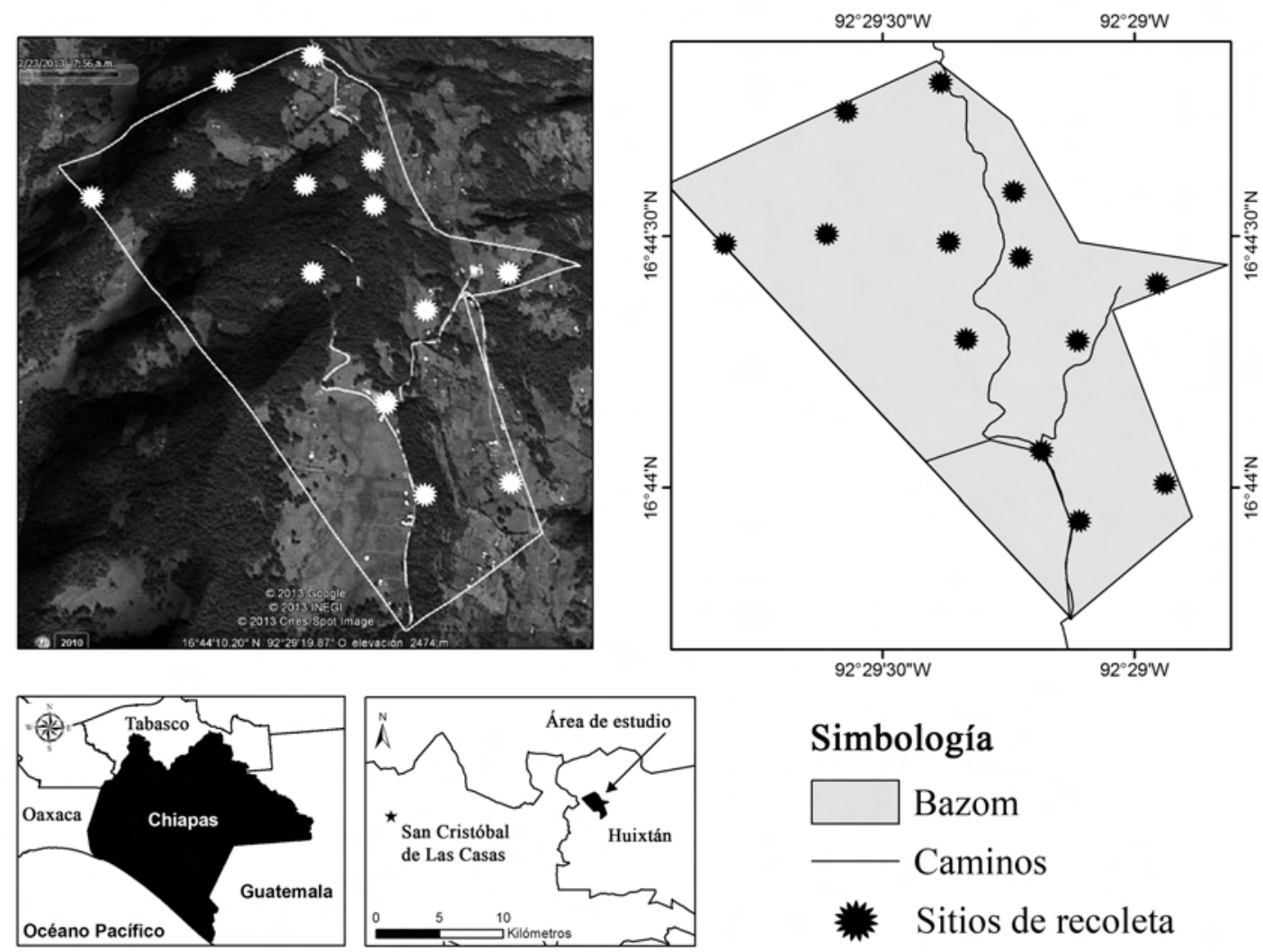

Simbología

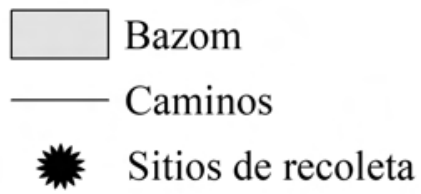

Figura 1. Área de estudio y localidades muestreadas. 
trabaje las parcelas, por lo que la superficie de tierras que permanecen bajo cultivo es menor que en años anteriores. Esto ha ocasionado que, en los últimos años, se observen más terrenos abandonados, lo cual ha propiciado un proceso de restauración pasiva del bosque.

El objetivo de esta investigación fue inventariar la flora vascular en un bosque de pino-encino con diferentes categorías de disturbio, ubicado en la comunidad de Bazom, en el estado de Chiapas, México.

\section{Materiales y métodos}

Área de estudio. La comunidad de Bazom se localiza a $16 \mathrm{~km}$ al este de San Cristóbal de Las Casas, en el municipio de Huixtán ( $16^{\circ} 44^{\prime} 50^{\prime \prime} \mathrm{N}$ y $\left.92^{\circ} 29^{\prime} 45^{\prime \prime} \mathrm{O}\right)$ entre 2,400 y 2,500 m de altitud (Figura 1). La vegetación incluye un mosaico heterogéneo de comunidades sucesionales del bosque de pinoencino (González-Espinosa et al., 1991, 2007).

Comunidades vegetales. Las comunidades vegetales donde se encuentran las especies registradas se definieron con base en los estudios realizados por González-Espinosa et al. (1991) y se dividieron en seis categorías: Campo abandonado (CA), área abandonada después de tres o cuatro años de haber sembrado la milpa (ocasionalmente pastoreado por ovejas). Pastizal (PA), pastoreado por ovejas y no se sabe con exactitud cuándo fue la última vez que se sembró la milpa. Matorral (MA), pastoreado por ovejas y con al menos tres capas de vegetación. Bosque temprano (BT), árboles de 10 a 15 m de altura y con pinos de 20 a 25 años de edad, el follaje más alto genera de 80 a $90 \%$ de sombra (existe una pequeña cantidad de epífitas y trepadoras) sobre las ramas de los árboles más grandes; en este tipo de vegetación se colectan polines y leña. Bosque mediano (BME), los pinos más grandes pueden tener de 40 a 45 años de edad, a una altura de 20 a $25 \mathrm{~m}$, el follaje genera de 70 a $80 \%$ de sombra, la mayoría de las ramas grandes de los árboles están cubiertas por epífitas y trepadoras; en este tipo de vegetación ocasionalmente se colectan pequeños polines, leña y tablas largas. Bosque maduro (BM), los pinos más grandes tienen una edad promedio de 80 a 100 años, se presentan dos capas de árboles dentro del bosque, una de 35 a $45 \mathrm{~m}$ y otra de 8 a 12 m; existe una gran cantidad de epífitas y trepadoras (Figura 2).

Recoletas botánicas. Las recolectas botánicas se realizaron durante 12 salidas de campo (junio 2012-febrero 2013), con una duración aproximada de ocho horas cada una, haciendo recorridos en los caminos y senderos de las diferentes comunidades vegetales del bosque de pino-encino, no se consideraron en el muestreo especies cultivadas. Se recolectaron 334 muestras, 295 colecciones corresponden a Miguel Martínez-Icó (MAI) y 39 a William Cetzal-Ix (CET; Apéndice 1). Adicionalmente, se realizó una revisión de ejemplares depositados en el herbario ECOSUR (CH). La herborización de los ejemplares botánicos se realizó de acuerdo con la propuesta de Lot y Chiang (1986). Las determinaciones botánicas se realizaron en el herbario $\mathrm{CH}$ durante el 20122013. Se utilizó literatura especializada y claves taxonómicas (Breedlove, 1981; Mickel y Smith, 2004). En el caso de la determinación de especies de Pinus y Quercus se consultó: González-Villarreal (1986), Farjon y Styles (1997), Valencia (2002) y Ramírez-Marcial et al. (2010, 2012). Para las Gimnospermas y Pteridofitas se siguieron las propuestas de clasificación de Christenhusz et al. (2011a, b). Los especímenes recolectados se depositaron en el herbario $\mathrm{CH}$ y se enviaron duplicados de algunos helechos y orquídeas al herbario CICY (Apéndice 1).

El sistema de clasificación para la vegetación utilizado fue de acuerdo a Rzedowski (1978) y González-Espinosa et al. (1991). La sistemática de las familias está ordenada de acuerdo con Angiosperm Phylogeny Group (APG III; Chase y Reveal, 2009) y dentro de las subclases fueron ordenadas alfabéticamente. Los estados de conservación indicados para las especies son los citados en la NOM-059SEMARNAT-2010 y en The Red List of Mexican Cloud Forest Trees (González-Espinosa et al., 2011), basado en los criterios de la IUCN (2012).

Hábito y formas de vida. Las especies se clasificaron en cuatro hábitos de crecimiento: árboles, arbustos, hierbas y trepadoras. Se registraron las formas de vida terrestre o epífita para las familias de la subclase Polypodiidae y las familias Bromeliaceae, Orchidaceae y Piperaceae.

Nombres comunes y usos: Se incluye para las especies, que así lo presentan, su nombre común y su respectivo uso en la comunidad y zonas adyacentes. Miguel Martínez Icó, originario de Bazom y hablante de la lengua tzotzil, proporcionó la mayoría de los nombres comunes y usos de las plantas; además, se consultó literatura especializada (Berlin et al., 1974).

\section{Resultados}

Se registraron un total de 353 taxa, repartidos en 342 especies, tres subespecies, siete variedades y un híbrido natural, agrupados en 95 familias y 235 géneros (Cuadro 1, Apéndice 1).

La familia con mayor número de taxa fue Asteraceae con 57, seguido de Poaceae con 17, Solanaceae con 14, Rosaceae con 14, Orchidaceae con 13, Polypodiaceae y Fabaceae con diez cada una, Rubiaceae y Lamiaceae con nueve cada una y, Onagraceae con ocho. En estas diez familias están representadas el 46\% (161) de los taxa registrados en el inventario florístico. Las familias con mayor número de géneros fueron Asteraceae con 39, Poaceae con 16 y Orchidaceae con diez. Los géneros con mayor número de especies son Solanum 
Miguel MartíneZ-Icó ET AL.
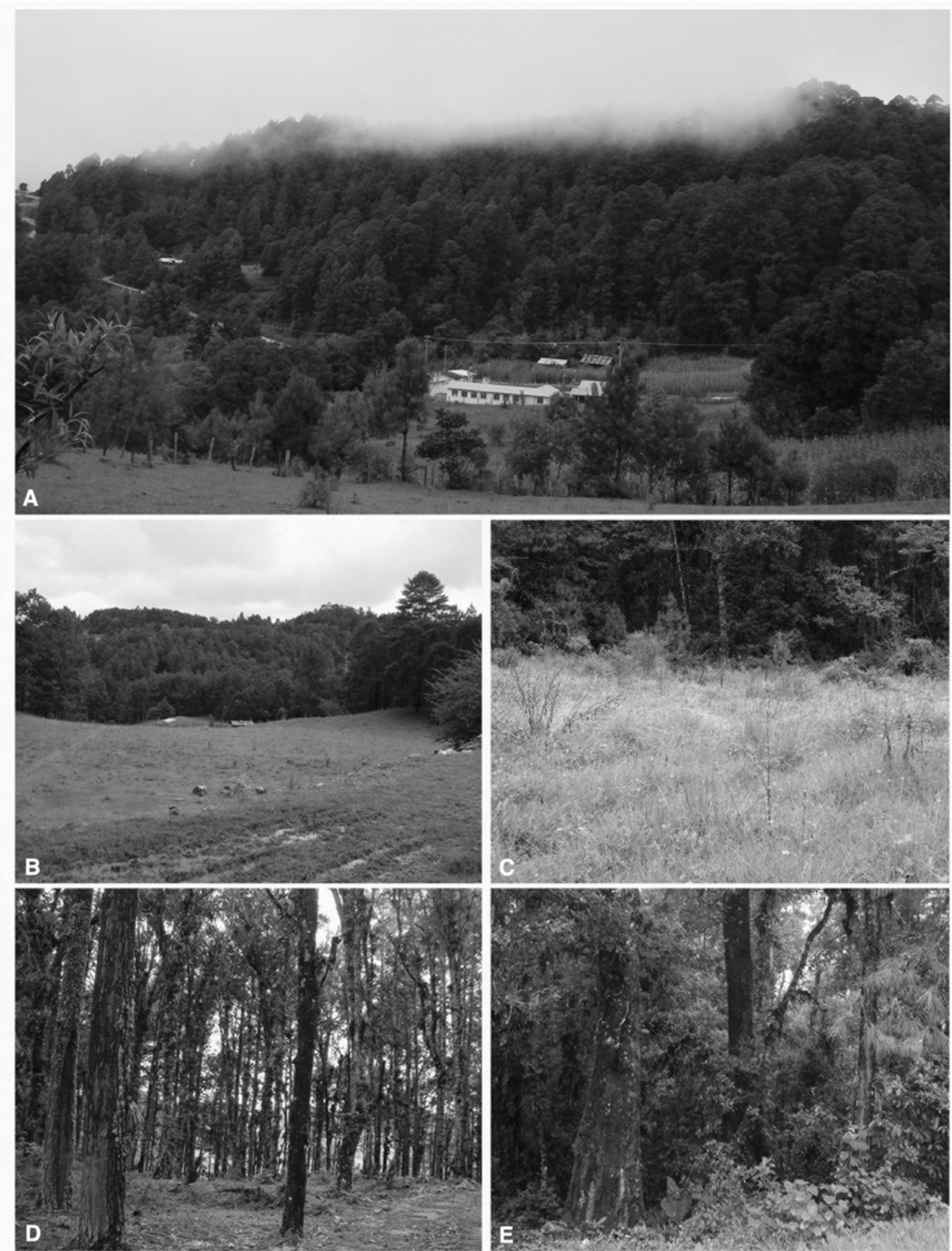

Figura 2. Tipos de vegetación de la comunidad de Bazom. A) bosque de pino-encino, B) campo abandonado, C) pastizal, D) bosque temprano, E) bosque maduro. 
Flora vascular de BAZOM, ChiAPAS

Cuadro 1. Comparación de datos cuantitativos de la diversidad florística entre la comunidad de Bazom y La Reserva Ecológica Cerro Huitepec, Chiapas, México.

\begin{tabular}{|c|c|c|c|c|c|c|}
\hline \multicolumn{7}{|c|}{ Este estudio } \\
\hline Subclase & Familia & Género & Especie & Subespecie & Variedad & Híbrido \\
\hline Lycopodiidae & 2 & 3 & 3 & - & - & - \\
\hline Equisetidae & 1 & 1 & 1 & - & - & - \\
\hline Marattiidae & - & - & - & - & - & - \\
\hline Polypodiidae & 12 & 23 & 38 & 1 & 1 & - \\
\hline Pinidae & 2 & 2 & 5 & - & 3 & - \\
\hline Magnoliidae & 78 & 207 & 295 & 2 & 3 & 1 \\
\hline Total & 95 & 235 & 342 & 3 & 7 & 1 \\
\hline \multicolumn{7}{|c|}{ Ramírez-Marcial et al. (1998)** } \\
\hline Lycopodiidae & 1 & 2 & 2 & - & - & - \\
\hline Equisetidae & - & - & - & - & - & - \\
\hline Marattiidae & 1 & 1 & 1 & - & - & - \\
\hline Polypodiidae & 12 & 18 & 31 & 1 & 2 & - \\
\hline Pinidae & - & - & - & - & - & - \\
\hline Magnoliidae & 72 & 164 & 242 & 9 & 10 & - \\
\hline Total & 86 & 185 & 276 & 10 & 12 & - \\
\hline
\end{tabular}

** (17 taxa registrados hasta familia o género).

con siete, seguido de Tillandsia con seis, Asplenium, Fuchsia, Quercus y Smilax con cinco especies cada uno. Para 81 especies se citan los usos dados en la comunidad y se incluyen nombres en lengua tzotzil con los que la mayoría son identificadas en la zona de Los Altos de Chiapas y, también algunos nombres comunes en castellano.

Se consideraron cuatro hábitos de crecimiento, de los cuales el más abundante fue las hierbas, seguidas de los arbustos, árboles y trepadoras, con 225, 66, 47 y 15 respectivamente. La familia más abundante con el hábito de crecimiento hierba fue la Asteraceae con 36 especies, seguido por las Poaceae con 17; algunas de estas especies con amplia distribución geográfica en el Neotrópico, otras con distribución cosmopolita como: Conyza bonariensis, Galinsoga quadriradiata, Gnaphalium americanum, Poa апnиa, Sonchus asper, Sporobolus indicus, Taraxacum officinale y Vulpia bromoides. En conjunto, las familias Asteraceae,
Orchidaceae, Poaceae y Polypodiaceae aportan el 34\% del componente herbáceo muestreado.

El dosel del bosque maduro está dominado por Pinus ayacahuite var. ayacahuite, P. montezumae var. montezumae, $P$. pseudostrobus, $P$. pseudostrobus var. apulcensis, $P$. tecunumanii, P. maximinoi, P. oocarpa, Quercus benthamii, $Q$. crassifolia, $Q$. crispipilis, $Q$. laurina y $Q$. rugosa, todos estos taxa son de alta importancia económica por ser fuente de madera, leña y carbón en la comunidad de Bazom. Entre las especies epífitas abundantes en el bosque maduro se encuentran Tillandsia guatemalensis y T. lautneri; por el contrario, en el bosque temprano abundan Tillandsia ponderosa, todas creciendo sobre Pinus spp.

Un total de 55 especies se encuentran bajo alguna categoría de riesgo de acuerdo a la IUCN y la NOM059- SEMARNAT-2010 (Cuadro 2). De las 47 especies de árboles presentes en el área de estudio, 37 están bajo algún

Cuadro 2. Datos cuantitativos de especies consideradas en alguna categoría de riesgo por la IUCN y la NOM-059-SEMARNAT-2010. The red list of Mexican cloud forest trees: $\mathrm{Vu}=$ vulnerable, $\mathrm{En}=$ en peligro, $\mathrm{CR}=$ en peligro crítico, $\mathrm{NT}=$ casi amenazado, $\mathrm{LC}=$ preocupación menor NOM-059 SEMARNAT-2010: $\operatorname{Pr}=$ sujetas a protección especial, A = amenazadas.

\begin{tabular}{llccccc}
\hline & Categoría & Hierbas & Trepadoras & Arbustos & Árboles & Total \\
\hline Vu & Vulnerable & - & - & 4 & 12 & 16 \\
En & En peligro & - & - & 3 & 7 & 10 \\
CR & En peligro crítico & - & - & - & 5 & 1 \\
NT & Casi amenazado & - & - & - & 12 & 7 \\
LC & Preocupación menor & - & - & - & - & 18 \\
& Total & - & - & - & - & 52 \\
\hline Pr & Sujetas a protección especial & 2 & - & - & - & 1 \\
A & Amenazadas & 1 & - & & 37 \\
& Total & 3 & & & - & 3 \\
\hline
\end{tabular}


riesgo, lo que representa el $79 \%$ de las especies arbóreas de Bazom; de estas, Oreopanax arcanus, que tiene distribución en la Altiplanicie Central, la Sierra Madre de Chiapas, México, y en Huehuetenango, Guatemala (González-Espinosa et al., 2011), se encuentra en peligro crítico (CR) de conservación por pérdida del hábitat. En Bazom, los individuos observados fueron escasos y aislados, generalmente con un diámetro aproximado de $20 \mathrm{~cm}$, creciendo como árboles medianos en el interior del bosque. En la categoría de peligro (EN) se encuentran siete especies arbóreas: Bocconia gracilis, Magnolia sharpii, Miconia hemenostigma, Olmediella betschleriana, Persea americana, Quercus benthamii y Viburnum acutifolium.

En el bosque maduro se registró el mayor número de especies (208), siendo las hierbas el grupo más diverso (108 spp.), en segundo lugar el pastizal con 98, donde evidentemente también las hierbas ( $88 \mathrm{spp}$.) son la mayoría (Cuadro 3). El bosque mediano, en tercer lugar, con 94 especies, y con valores muy similares el campo abandonado con 80 especies; en ambos tipos de vegetación, con una alta diversidad de hierbas, 50 y 69, respectivamente. En todos los tipos de vegetación muestreados los árboles representan el grupo menos diverso, después de las trepadoras (Cuadro 3).

\section{Discusión}

La riqueza registrada para Bazom (353 taxa), constituye un aporte al conocimiento de la flora de Los Altos de Chiapas, y además, le confiere importancia ecológica como un remanente de los bosques de pino-encino en el estado. Resultados similares fueron registrados en Los Altos de Chiapas por Ramírez-Marcial et al. (1998), al inventariar 315 especies en el bosque de encino en la Estación Biológica Cerro Huitepec (EBCH), San Cristóbal de Las Casas, Chiapas (Cuadro 1). En general, estos bosques de montaña son los ecosistemas forestales más seriamente amenazados por actividades humanas, por lo que su conservación, manejo y restauración son temas de interés nacional e internacional (Budd et al., 2004). En México, los bosques de pino-encino se encuentran entre los tipos de vegetación más afectados por el asentamiento y las actividades de subsistencia del ser humano, debido a la fertilidad de sus suelos, su agradable

Cuadro 3. Datos cuantitativos de los hábitos de crecimiento en las comunidades de vegetación. $\mathrm{BM}=$ bosque maduro, $\mathrm{BME}=$ bosque mediano, $\mathrm{BT}=$ bosque temprano, $\mathrm{CA}=$ campo abandonado, $\mathrm{MA}=$ matorral, $\mathrm{PA}=$ pastizal.

\begin{tabular}{lcccccc}
\hline Hábito & BM & BME & BT & CA & MA & PA \\
\hline Árboles & 42 & 15 & 14 & 1 & 3 & 1 \\
Arbustos & 46 & 26 & 21 & 8 & 16 & 9 \\
Hierbas & 108 & 50 & 37 & 69 & 44 & 88 \\
Trepadoras & 12 & 3 & 3 & 2 & 2 & - \\
Total & 208 & 94 & 75 & 80 & 65 & 98 \\
\hline
\end{tabular}

clima y sus estaciones bien definidas. Como resultado de ello, hace mucho tiempo que se empezaron a destruir estos bosques en vastas áreas de su distribución natural (Rzedowski, 1978; Ramírez-Marcial et al., 2001; González-Espinosa et al., 2008); por lo que los fragmentos de bosque en la comunidad de Bazom y en $\mathrm{EBCH}$, representan parte de los últimos remanentes de bosque en los Altos de Chiapas.

De acuerdo con Ramírez-Marcial et al. (2001) y OchoaGaona et al. (2004), junto con la deforestación, la fragmentación y el aislamiento de los rodales, se ha producido un empobrecimiento florístico de los bosques secundarios. La tendencia observada en bosques previamente dominados por especies de Quercus ha sido el incremento de especies de Pinus, asociado a una reducción en la riqueza de trepadoras, arbustos y árboles del interior. Tal como se registró en Bazom donde el número de especies de Pinus supera al de Quercus, y la diversidad de especies trepadoras y otras formas de vida fue muy bajo. Esta condición, podría también estar favorecida por el comportamiento ecológico de los pinos, que en su mayoría son resistentes a las heladas, a largos períodos de sequía, incendios fuertes y al pastoreo; además de establecerse sobre suelos someros, rocosos y muchas veces pobres en nutrientes y minerales, características que les confieren capacidad de invadir fácilmente sitios más favorables y prosperar en ellos, aunque, de no persistir las circunstancias propicias, pueden sucumbir a la larga latencia ante la competencia de otras asociaciones vegetales mejor adaptadas (Rzedowski, 2006). Ejemplo de esto es Pinus oocarpa, especie al parecer resistente y muy favorecida por los fuegos, ya que presenta la característica de conos serótinos, que por la acción de altas temperaturas se abren y permiten la liberación simultánea de grandes cantidades de semillas (Rzedowski, 2006).

De las proporciones de cobertura que en un determinado lugar guarden entre sí los componentes de un bosque mixto de Pinus y Quercus, dependerá mucho su aspecto general, su comportamiento estacional, así como la composición y estructura de los estratos inferiores (Rzedowski, 2006). En Bazom los estratos inferiores estuvieron dominados por las hierbas, el hábito de mayor diversidad específica, en su mayoría perteneciente a Asteraceae. Coincidiendo con lo señalado por Rzedowski (2006), el espectro biológico de los pinares indica una fuerte preponderancia de los elementos herbáceos sobre los leñosos, y la familia Asteraceae (Compositae) suele estar muy bien representada, además de las gramíneas; este patrón también se observó por RamírezMarcial et al. (1998) en la EBCH. Sin embargo, además de las Asteraceae y las Poaceae, son frecuentes en Los Altos de Chiapas, y asociadas al bosque de pino y pino-encino, las familias Solanaceae y Rosaceae, representadas por una amplia diversidad de especies herbáceas (Figura 3) y arbustivas. A semejanza de la EBCH, en Bazom, las hierbas perennes y anuales estuvieron presentes en todas las comunidades vegetales muestreadas. 

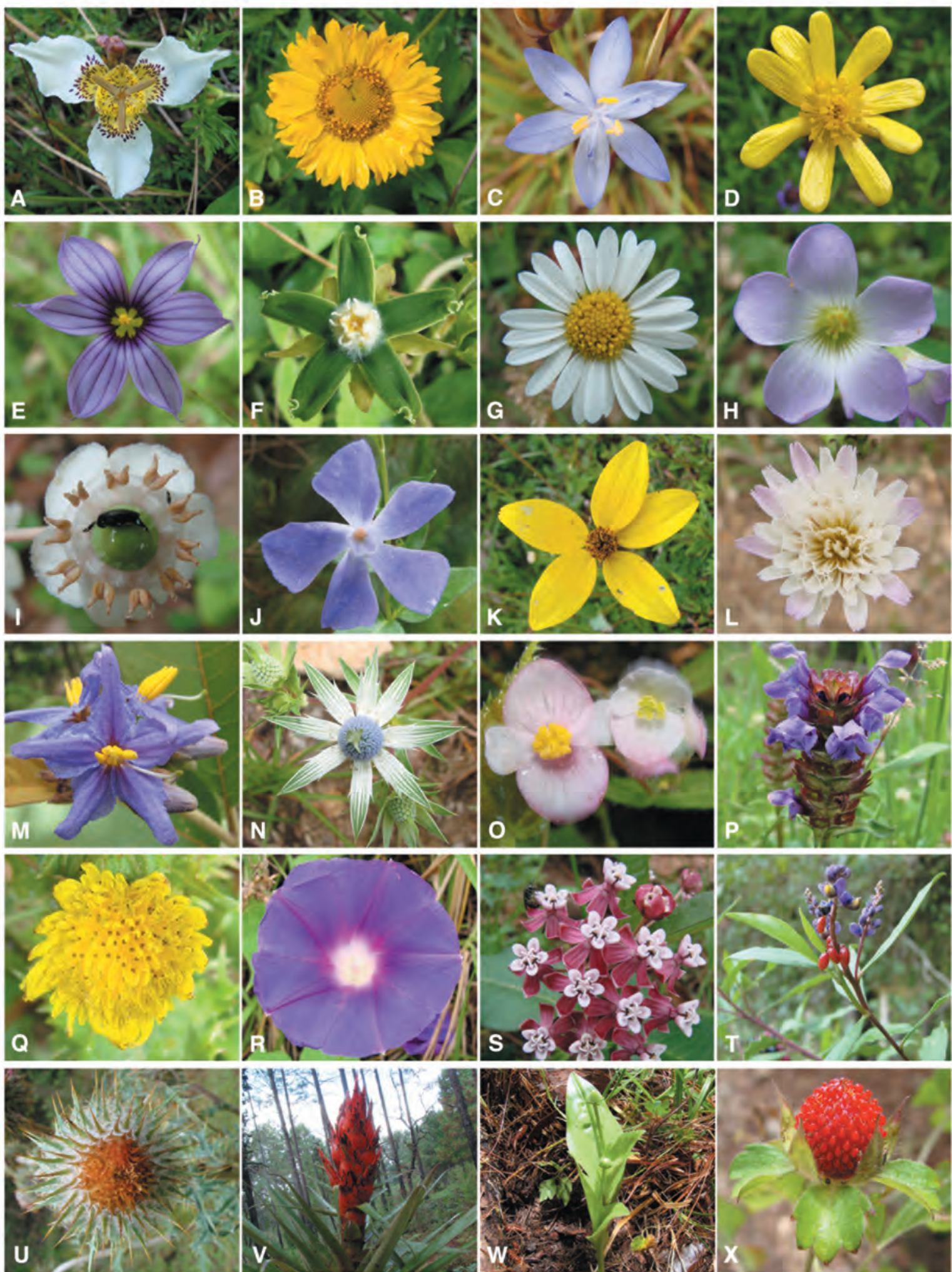

Figura 3. Representantes de la flora de la comunidad de Bazom. A) Tigridia chiapensis (endémica), B) Helenium scorzoneraefolium, C) Commelina diffusa, D) Ranunculus petiolaris, E) Sisyrinchium scabrum, F) Gonolobus stenosepalus, G) Symphyotrichum moranense, H) Oxalis alpina, I) Chimaphila maculata, J) Vinca major, K) Bidens triplinervia, L) Trifolium repens, M) Solanum lanceolatum, N) Eryngium scaposum, O) Begonia oaxacana, P) Prunella vulgaris, Q) Sonchus asper, R) Ipomoea purpurea. S) Asclepias similis, T) Monina xalapensis, U) Cirsium subcoriaceum, V) Tillandsia ponderosa, W) Malaxis brachyrrhynchos, X) Fragaria vesca. 
En Chiapas, al igual que en la Sierra Madre del Sur, los pinares de Pinus oocarpa son los más difundidos, y su amplitud altitudinal va de 300 a 3,000 m; por otra parte, las especies $P$. ayacahuite, $P$. montezumae y $P$. pseudostrobus están presentes en sitios muy húmedos (Miranda, 1952), siendo estas algunas de las especies de Pinus, registradas en este estudio y que en conjunción con Quercus spp., particularmente $Q$. laurina, dominan el dosel del bosque en Bazom. Quercus y Pinus son elementos de afinidad holártica, que se pueden establecer en etapas serales tempranas e incluso migrar a través de áreas no arboladas relativamente secas (Quintana-Ascencio y González-Espinosa, 1993).

La diversidad de árboles registrados en Bazom se encuentra entre los valores esperados, de acuerdo a GonzálezEspinosa et al. (2009): un fragmento moderadamente bien conservado de bosque mesófilo de montaña o de bosque de pino-encino contiene 40-50 especies arbóreas por hectárea. Entre las especies de importancia por su estado de conservación, Magnolia sharpii y Persea americana son abundantes en el bosque maduro, con poblaciones jóvenes y plántulas observadas durante el desarrollo del inventario. En el caso de Persea americana, a pesar de la gran variedad de cultivares desarrollados a través del tiempo y lo ampliamente cultivada en América Central, las poblaciones silvestres de esta especie se encuentran en peligro crítico, debido a la perturbación de su hábitat, restringido a bosques nublados, bosques húmedos de pino-encino y bosque de encino, así como también al bosque semi-siempreverde tropical. Otras especies en peligro, como Bocconia gracilis, tiene distribución restringida a Chiapas, y Quercus benthamii y Viburnum acutifolium se distribuyen solo en bosques nublados húmedos de Oaxaca y Chiapas, lo que las hace más susceptibles a desaparecer.

La composición de Pteridofitas, inventariadas por Ramírez-Marcial et al. (1998), presenta amplias similitudes en la composición de familias, géneros y especies registradas en Bazom (Apéndice 1), en ambas localidades el género más diverso fue Asplenium. Por otra parte, en Bazom, en el bosque maduro, la abundancia en el sotobosque de Polypodium representa un indicador de la humedad del ambiente y de su buen estado de conservación. Estas condiciones de humedad, conservación y abundancia de hojarasca han favorecido también, en el bosque maduro, el desarrollo de orquídeas terrestres, llegando a ser más numerosas en diversidad que las orquídeas epífitas. Las especies más abundantes del sotobosque son Govenia superba y Liparis arnoglossophylla.

González-Espinosa et al. (2008) propusieron que la riqueza florística remanente en los fragmentos forestales derivados del patrón tradicional de uso del suelo, representa un potencial para diseñar prácticas de restauración forestal con el uso de un elevado número de especies arbóreas nativas, de aquí la importancia del inventario de la flora de estos fragmentos de bosque.
Por otra parte, en las últimas décadas, estudiosos de múltiples disciplinas han insistido en la asociación de la pérdida de especies biológicas, de la funcionalidad de los ecosistemas y de la reducción de la capacidad para generar servicios ambientales básicos, con el deterioro de las culturas y los grupos lingüísticos (Boege, 2008). Si bien varios autores se han referido a la pérdida de la diversidad biológica (Wilson, 1989; Boege, 2008), es menos conocida la relación entre la pérdida de las especies y el deterioro de las lenguas y las culturas del mundo, con lo que resulta de importancia el conocimiento de estas floras asociadas a comunidades indígenas, además de la inclusión y difusión de los nombres comunes de las plantas designados en lenguas indígenas. De aquí la importancia de la inclusión de los nombres en tzotzil y el uso de algunas de las especies presentes en Bazom y áreas adyacentes. Este conocimiento comunitario de la flora y su reconocimiento por medio de nombres comunes, constituyen un aspecto importante para el fomento de la conservación de las especies, particularmente las de mayor uso.

El conocimiento y la conservación de la flora de Los Altos de Chiapas sigue siendo una necesidad, y en estos tiempos demanda urgencia debido a la acelerada fragmentación que ha sufrido ante el cambio de uso del suelo.

\section{Agradecimientos}

Los autores agradecen a Neptalí Ramírez Marcial y Mario González Espinosa (ECOSUR-SCL) por las facilidades otorgadas para realizar este trabajo. El segundo autor agradece al CONACYT por las becas otorgadas para estudios doctorales y posdoctorales. El tercer autor agradece al ECOSUR-SCL por la beca otorgada para estudios posdoctorales. A Isabel Vásquez Lara y Mario Ishiki Ishihara (ECOSURSCL) por las facilidades otorgadas para la revisión de la base de datos e identificación de los ejemplares en el herbario de ECOSUR (CH). A Gustavo Romero González (AMES) por sus comentarios y sugerencias al resumen en inglés. A Juan Pablo Pinzón Esquivel quien identificó a Tillandsia lautneri. Agradecemos a María Hilda Flores Olvera y un revisor anónimo por sus valiosos comentarios al manuscrito. A Silvia Hernández Aguilar (CICY) por su colaboración en el etiquetado de los especímenes de helechos y orquídeas depositados en el herbario CICY.

\section{Literatura citada}

Berlin B., Breedlove D.E. y Raven P.H. 1974. Principles of Tzeltal Plant Classification: An Introduction to the Botanical Ethnography of a Mayan-Speaking People of Highland Chiapas. Academic Press, Nueva York.

Boege E. 2008. El Patrimonio Biocultural de los Pueblos Indígenas de México. Hacia la Conservación in situ de la Biodiversidad y Agrodiversidad en los Territorios Indígenas. Instituto Nacional de Antropología e Historia, Comisión Nacional para el Desarrollo de los Pueblos Indígenas, México, D.F. 
Breedlove D.E. 1981. Introduction to the Flora of Chiapas. Part I. California Academy of Science, San Francisco.

Budd P., May I., Miles L. y Sayer J. 2004. Cloud Forest Agenda. 〈http://www.ourplanet.com/wcmc/pdfs/cloudforests.pdf> (consultado 5 junio 2012).

Chase M.W y Reveal J.L. 2009. A phylogenetic classification of the land plants to accompany APG III. Botanical Journal of the Linnean Society 161:122-127.

Christenhusz M.J.M., Reveal J.L., Farjon A., Gardner M.F., Mill R.R. y Chase M.W. 2011a. A new classification and linear sequence of extant gymnosperms. Phytotaxa 19:55-70.

Christenhusz M.J.M., Zhang X.C. y Schneider H. 2011b. A linear sequence of extant families and genera of lycophytes and ferns. Phytotaxa 19:7-54.

Farjon A. y Styles B.T. 1997. Pinus (Pinaceae). Flora Neotropica Monograph 75:1-291.

Galindo-Jaimes L., González-Espinosa M., Quintana-Ascencio P. y García-Barrios L. 2002. Tree composition and structure in disturbed stands with varying dominance by Pinus spp. in the highlands of Chiapas, Mexico. Plant Ecology 62:259-272.

González-Espinosa M., Ocho-Gaona S., Ramírez-Marcial N. y Quintana-Ascencio P.F. 1997. Contexto vegetacional y florístico de la agricultura. En: Parra-Vázquez M.R. y Díaz-Hernández B.M. Eds. Los Altos de Chiapas: Agricultura y Crisis Rural. Tomo I. Los Recursos Naturales, pp. 85-117, El Colegio de la Frontera Sur, San Cristóbal de las Casas.

González-Espinosa M., Quintana-Ascencio P.F., Ramírez-Marcial N. y Gaytán-Guzmán P. 1991. Secondary succession in disturbed Pinus-Quercus forests in the highlands of Chiapas, Mexico. Journal of Vegetation Science 2:351-360.

González-Espinosa M., Ramírez N., Quintana P.F. y Martínez M. 1995. La utilización de encinos y la conservación de la biodiversidad en Los Altos de Chiapas. Memorias del Tercer Seminario Nacional sobre Utilización de Encinos. Reporte Científico UANL 15(Núm.Esp.):183-197.

González-Espinosa M., Ramírez-Marcial N., Camacho-Cruz A. y Rey-Benayas J.M. 2008. Restauración de bosques en montañas tropicales de territorios indígenas de Chiapas, México. En: González-Espinosa M., Rey-Benayas J.M. y Ramírez-Marcial N. Eds. Restauración de Bosques en América Latina, pp. 137162, Fundación Internacional para la Restauración de Ecosistemas, Editorial Mundi-Prensa, México, D.F.

González-Espinosa M., Meave J.A., Lorea-Hernández F.G., Ibarra-Manríquez G. y Newton A.C. Eds. 2011. The Red List of Mexican Cloud Forest Trees. Fauna and Flora International, Cambridge.

González-Espinosa M., Ramírez-Marcial N., Méndez-Dewar G., Galindo-Jaimes L. y Golicher D. 2005. Riqueza de especies de árboles en Chiapas: variación espacial y dimensiones ambientales asociadas al nivel regional. En: González-Espinosa M., Ramírez-Marcial N. y Ruiz-Montoya L. Coords. Diversidad Biológica de Chiapas, pp. 81-125, Consejo de Ciencia y Tecnología del Estado de Chiapas, El Colegio de la Frontera Sur, Plaza y Valdés, México, D.F.

González-Espinosa M., Ramírez-Marcial N., Camacho-Cruz A., Holz S.C., Rey-Benayas J.M. y Parra-Vázquez M.R. 2007. Restauración de bosques en territorios indígenas de Chiapas: modelos ecológicos y estrategias de acción. Boletín de la Sociedad Botánica de México 80(Supl.):11-23.

González-Espinosa M., Ramírez-Marcial N., Galindo-Jaimes L.,
Camacho-Cruz A., Golicher D., Cayuela L. y Rey-Benayas J.M. 2009. Tendencias y proyecciones del uso del suelo y la diversidad florística de Los Altos de Chiapas. Investigación Ambiental Ciencia y política pública 1:40-53.

González-Villarreal L.M. 1986. Contribución al conocimiento del género Quercus (Fagaceae) en el Estado de Jalisco. Flora de Jalisco 1:1-240.

IUCN. Internacional Union for Conservation of Nature. 2012. Categorías y Criterios de la Lista Roja de la IUCN. Versión 3.1. $2^{\mathrm{a}}$ ed. Internacional Union for Conservation of Nature, Gland.

Lot A. y Chiang C.F. 1986. Manual de Herbario. Administración y Manejo de Colecciones, Técnicas de Recolección y Preparación de Ejemplares Botánicos. Consejo Nacional de la Flora de México, A.C., México, D.F.

Mickel J.T. y Smith A.R. 2004. The Pteridophytes of Mexico. Memoirs of the New York Botanical Garden 88:1-1054.

Miranda F. 1952. La vegetación de Chiapas, vol. 1. Ediciones del Gobierno del Estado, Tuxtla Gutiérrez.

Ochoa-Gaona S., González-Espinosa M., Meave J.A. y Sorani-Dal Bon V. 2004. Effect of forest fragmentation on the woody flora of the highlands of Chiapas, Mexico. Biodiversity and Conservation 13:867-884.

Quintana-Ascencio P.F. y González-Espinosa M. 1993. Afinidad fitogeográfica y papel sucesional de la flora leñosa de los bosques de Pino-encino de los Altos de Chiapas, México. Acta Botanica Mexicana 21:43-57.

Palacio-Prieto J.L., Bocco G., Velázquez A., Mas J.F., TakakiTakaki F., Victoria A., Luna-González L., Gómez-Rodríguez G., López-García J., Palma M.M., Trejo-Vázquez I., Peralta H.A., Prado-Molina J., Rodríguez-Aguilar A., Mayorga-Saucedo R. y González M.F. 2000. La condición actual de los recursos forestales de México: resultados del Inventario Forestal Nacional 2000. Investigaciones Geográficas 43:183-203.

Parra-Vázquez M.R., Nahed-Toral J., Soto-Pinto M.L., GarcíaAguilar M.C. y García-Barrios L.E. 1993. El sistema ovino tzotzil de Chiapas: I. Dinámica del manejo integral. Agrociencia. Serie Recursos Naturales Renovables 3:79-97.

PEOT. Programa Estatal de Ordenamiento Territorial del Estado de Chiapas. 2005. Programa de ordenamiento territorial del Estado de Chiapas (fases I y II). Anexo 1. Matriz de cambios. Cambio de vegetación y uso de suelo 1975-2000. Secretaría de Planeación y Finanzas, Gobierno del Estado de Chiapas, Tuxtla Gutiérrez.

Pérez-Farrera M.A., Martínez-Camilo R., Martínez-Meléndez N., Farrera-Sarmiento O. y Villalobos-Méndez S. 2012. Listado florístico del Cerro Quetzal (Polígono III) de la reserva de la biosfera El Triunfo, Chiapas, México. Botanical Sciences 90:113-142.

Ramírez-Marcial N., González-Espinosa M. y Williams-Linera G. 2001. Anthropogenic disturbance and tree diversity in montane rain forest in Chiapas, Mexico. Forest Ecology and Management 154:311-326.

Ramírez-Marcial N., Camacho-Cruz A., Martínez-Icó M., LunaGómez A., Golicher D. y González-Espinosa M. 2010. Árboles $y$ arbustos de los bosques de montaña en Chiapas. El Colegio de la Frontera Sur, San Cristóbal de las Casas.

Ramírez-Marcial N., Luna-Gómez A., Castañeda O.H.E., Martínez-Icó M., Holz S.C., Camacho C.A. y González-Espinosa M. 2012. Guía de Propagación de Árboles Nativos para la Recuperación de Bosques. $2^{\mathrm{a}}$ ed. El Colegio de la Frontera Sur, San Cristóbal de las Casas. 
Ramírez-Marcial N., Ochoa-Gaona S., González-Espinosa M. y Quintana-Ascencio P.F. 1998. Análisis florístico y sucesional en la estación biológica Cerro Huitepec, Chiapas, México. Acta Botanica Mexicana 44:59-85.

Ramos H.S. 2013. Suelos: Conocimiento y Problemática. En: Comisión Nacional para el Conocimiento y Uso de la Biodiversidad. La Biodiversidad en Chiapas: Estudio de Estado, Vol. I, pp. 41-51. Comisión Nacional para el Conocimiento y Uso de la Biodiversidad, Gobierno del Estado de Chiapas, México, D.F.

Rzedowski J. 1978. Vegetación de México. Limusa, México, D.F.

Rzedowski J. 1998. Diversidad y orígenes de la flora fanerogámica de México. En: Ramamoorthy T.P., Bye R., Lot A. y Fa J. Eds. Diversidad Biológica de México: Orígenes y Distribución, pp. 129-145, Instituto de Biología, Universidad Nacional Autónoma de México, México, D.F.

Recibido: 26 de septiembre de 2013 Aceptado: 20 de diciembre de 2013
Rzedowski J. 2006. Vegetación de México. $1^{\text {a }}$ Edición digital, Comisión Nacional para el Conocimiento y Uso de la Biodiversidad, México, D.F.

SEMARNAT. Secretaría del Medio Ambiente y Recursos Naturales. 2010. Norma Oficial Mexicana NOM-059-SEMARNAT2010 Protección ambiental-Especies nativas de México de flora y fauna silvestres-Categorías de riesgo y especificaciones para su inclusión, exclusión o cambio-Lista de especies en riesgo.

Valencia A.S., Gómez-Cárdenas M. y Becerra-Luna F. 2002. Catálogo de Encinos del Estado de Guerrero, México. Libro Técnico No. 1. Instituto Nacional de Investigaciones Forestales, Agrícolas y Pecuarias, México, D.F.

Wilson E.O. 1989. Threats to biodiversity. Scientific American 261:108-116. 
Apéndice 1. Listado de especies de la comunidad de Bazom, Chiapas. Colectores: Cetzal \& Martínez-lCó (CET-MAI), Cetzal, Noguera \& Martínez-Icó (CET et al.), González-Espinosa et al. (GOES), Martínez-lcó \& Cetzal (MAl-CET). Tipos de vegetación: bosque maduro (BM), bosque mediano (BME), bosque temprano (BT), campo abandonado (CA), matorral (MA), pastizal (PA). Formas de vida: terrestre, epífita. Categorías de conservación: $(*)$ Norma oficial mexicana NOM-059-SEMARNAT-2010: sujetas a protección especial $(\mathbf{P r})$, amenazadas $(\mathbf{A}) .\left(^{(*)}\right)$ The Red List of Mexican Cloud Forest Trees): vulnerable (VU), en peligro (En), en peligro crítico $(\mathbf{C R})$, casi amenazado (NT), preocupación menor $(\mathbf{L C})$.

\section{EQUISETOPSIDA}

\section{Subclase Lycopodiinae}

Lycopodiaceae

Lycopodium thyoides Humb. \& Bonpl. ex Willd. (CET-MAI $204(\mathrm{CH})$, hierba, BM).

Huperzia myrsinites (Lam.) Rothm. (CET et al. 280 (CH, CICY), hierba, BM).

\section{Selaginellaceae}

Selaginella apoda (L.) C.Morren (CET et al. $282(\mathrm{CH})$, hierba, BM).

\section{[Monilofitos]}

\section{Subclase Equisetidae}

Equisetaceae

Equisetum myriochaetum Schltdl. \& Cham. (MAI-CET $1161(\mathrm{CH})$, hierba, BM, medicinal y forrajera, Tut).

\section{Subclase Polypodiidae}

Aspleniaceae

Asplenium harpeodes Kunze (GOES $649(\mathrm{CH})$, hierba, BM).

Asplenium monanthes L. (CET-MAI 174, 200, 201 (CH, CICY), hierba, BM).

Asplenium praemorsum Sw. (CET-MAI $178(\mathrm{CH}, \mathrm{CICY})$, hierba, BM).

Asplenium serra Langsd. \& Fisch. (CET-MAl $179(\mathrm{CH}, \mathrm{CICY})$, hierba, BM).

Asplenium sessilifolium var. occidentale Desv. (CET-MAl $177(\mathrm{CH}, \mathrm{CICY})$, hierba, BM).

\section{Blechnaceae}

Blechnum polypodioides Raddi. (CET-MAI 203, 205 (CH, CICY), hierba, PA).

Woodwardia spinulosa M.Martens \& Galeotti (CET-MAI 167, 181, 209 (CH, CICY), hierba, BM).

\section{Cyatheaceae}

Cyathea fulva (M.Martens \& Galeotti) Fée (CET-MAl 166, $199(\mathrm{CH}, \mathrm{CICY})$, hierba, BM). (Pr)*

\section{Dennstaedtiaceae}

Pteridium aquilinum subsp. feei (W.Schaffn. ex Fée) J.A.Thomson, Mickel \& Mehltreter (CET-MAl 169 (CH, CICY), hierba, BM). Pteridium caudatum (L.) Maxon (CET-MAI $173(\mathrm{CH})$, hierba, BM).

\section{Dicksoniaceae}

Lophosoria quadripinnata (J.F.Gmel.) C.Chr. in Skottsb. (GOES 587 (CH), hierba, BM).

\section{Ophioglossaceae}

Botrychium decompositum M.Martens \& Galeotti (CET-MAI 176, $210(\mathrm{CH}, \mathrm{CICY})$, hierba, BM).

Ophioglossum vulgatum L. (GOES $1581(\mathrm{CH})$, hierba, BM).

\section{Dryopteridaceae}

Dryopteris wallichiana (Spreng.) Hyl. (CET-MAI 168 (CH, CICY), hierba, BM).

Elaphoglossum albomarginatum A.R.Sm. (CET-MAI 183 (CH, CICY), hierba, BM).

Elaphoglossum sartorii (Liebm.) Mickel (GOES $555(\mathrm{CH})$, hierba, BM).

Elaphoglossum vestitum (Schltdl. \& Cham.) T.Moore (Chamé \& Luna $219(\mathrm{CH})$, hierba, BM).

Elaphoglossum muscosum (Sw.) T.Moore (CET et al. $281(\mathrm{CH})$, hierba, BM).

\section{Lomariopsidaceae}

Lomariopsis recurvata Fée (GOES $566(\mathrm{CH})$, hierba, BM). 
Apéndice 1. Continuación

\section{Thelypteridaceae}

Macrothelypteris torresiana (Gaudich.) Ching (GOES $583(\mathrm{CH})$, hierba, BM).

Thelypteris kunthii (Desv.) C.V.Morton (CET-MAI 213 (CH), hierba, BME).

Thelypteris oaxacana A.R.Sm. (GOES $595(\mathrm{CH})$, hierba, BM).

Thelypteris oligocarpa (Humb.\& Bonpl. ex Willd.) Ching (GOES $588(\mathrm{CH})$, hierba, BM).

Thelypteris tetragona (Sw.) Small (GOES $579(\mathrm{CH})$, hierba, PA).

\section{Pteridaceae}

Adiantum andicola Liebm. (CET-MAI 172, 196, 207 (CH), hierba, BM/BME/BT).

Cheilanthes farinosa (Forssk.) Kaulf. (CET-MAI $194(\mathrm{CH})$, hierba, BT/CA/PA).

Cheilanthes pyramidalis Feé (CET-MAI $214(\mathrm{CH}, \mathrm{CICY})$, hierba, BT/CA).

Pteris cretica L. (CET-MAI $195(\mathrm{CH})$, hierba, BT).

Vittaria flavicosta Mickel \& Beitel (CET et al. $279(\mathrm{CH})$, hierba, BM).

\section{Polypodiaceae}

Campyloneurum amphostenon (Kunze ex Klotzsch) Fée (CET-MAl 175 ( $\mathrm{CH}, \mathrm{CICY})$, hierba, BM).

Campyloneurum angustifolium (Sw.) Fée (CET-MAl 197, $208(\mathrm{CH})$, hierba, BM).

Pecluma atra (A.M.Evans) M.G.Price (CET-MAI 206 (CH, CICY), hierba, BM).

Pleopeltis astrolepis (Liebm.) E.Fourn. (CET-MAI $184(\mathrm{CH})$, hierba, BM).

Pleopeltis mexicana (Fée) Mickel \& Beitel (CET-MAl $185(\mathrm{CH}, \mathrm{CICY})$, hierba, BM).

Polypodium plebeium Schltdl. \& Cham. (CET-MAI $180(\mathrm{CH}, \mathrm{CICY})$, hierba, BM).

Polypodium plesiosorum Kunze (CET-MAl 182, 185 (CH, CICY), hierba, BM).

Polypodium subpetiolatum Hook. (CET-MAI 170 (CH, CICY), hierba, BM).

Serpocaulon loriceum (L.) A.R.Sm. (GOES $581(\mathrm{CH})$, hierba, BM).

Serpocaulon triseriale (Sw.) A.R.Sm. (GOES $590(\mathrm{CH})$, hierba, BM).

\section{Woodsiaceae}

Athyrium arcuatum Liebm. (CET-MAl 187, 188 (CH, CICY), hierba, BM).

\section{[Gymnospermas]}

\section{Subclase Pinidae}

\section{Cupressaceae}

Cupressus lusitanica Mill. (MAl-CET 1153 (CH), árbol, CA, maderable y leña, Nukul pat).

\section{Pinaceae}

Pinus ayacahuite var. ayacahuite C.Ehrenb. ex Schltdl. (GOES $1885(\mathrm{CH})$, árbol, BM, maderable y leña, Temtoj, K'uktoj).

(VU A4ad; B1ab(i))**

Pinus montezumae var. montezumae Lamb. (MAI-CET $1214(\mathrm{CH})$, árbol, BM, maderable y leña, Botz-toj).

Pinus pseudostrobus Lindl. (MAI-CET $1193(\mathrm{CH})$, árbol, BM, maderable y leña, Sakil-Botz-toj). (LC)**

Pinus pseudostrobus var. apulcensis (Lindl.) Shaw (MAI-CET $1215(\mathrm{CH})$, árbol, BM, maderable y leña, Batz'i-toj). (LC)**

Pinus tecunumanii F.Schwerdtf. ex Eguiluz \& J.P.Perry (MAI-CET $1216(\mathrm{CH})$, árbol, BM/MA, maderable y leña,

Tzajal-shaktoj). (NT)**

Pinus maximinoi H.E.Moore (Martínez-lcó et al. $27(\mathrm{CH})$, árbol, BM).

Pinus oocarpa Schiede ex Schltdl. (GOES $1862(\mathrm{CH})$, árbol, BM).

\section{[Angiospermas]}

\section{Subclase Magnoliidae}

Actinidiaceae

Saurauia oreophila Hemsl. (MAI-CET $1183(\mathrm{CH})$, arbusto, BM/BME/BT, comestible (fruto), Cashlanté - Ajo'j). (VU B"ab(iii))**

\section{Adoxaceae}

Sambucus canadensis L. (MAI-CET $1103(\mathrm{CH})$, arbusto, CA/MA/PA).

Viburnum acutifolium Benth. (MAI-CET $1077(\mathrm{CH})$, arbusto, BME). (EN B1ab(ii)).

Viburnum elatum Benth. (MAl-CET $1247(\mathrm{CH})$, arbusto, BM/BME/BT, leña, Tsop). (VU B1ab(iii))**

Viburnum jucundum C.V.Morton (MAI-CET $1076(\mathrm{CH})$, árbol, BM/BME/BT, leña, Isbon Tsop). (EN B1 ab(iii))** 
Flora vascular DE BAZOM, Chiapas

Apéndice 1. Continuación

Alstroemeriaceae
Bomarea edulis (Tussac) Herb. (MAl-CET $1071(\mathrm{CH})$, hierba, BM, Yat-tz'i).

Amaranthaceae

Iresine diffusa Humb. \& Bonpl. ex Willd. (MAI-CET $1072(\mathrm{CH})$, hierba, BM, Pajal-itaj Womol).

\section{Amaryllidaceae}

Allium glandulosum Link \& Otto (MAI-CET $1170(\mathrm{CH})$, hierba, PA).

\section{Apiaceae}

Berula erecta (Huds.) Coville (MAI-CET 1050 (CH), hierba, BME).

Daucus montanus Humb. \& Bonpl. ex Spreng. (MAI-CET $1176(\mathrm{CH})$, hierba, CA/MA/PA).

Eryngium guatemalense Hemsl. (MAI-CET $1084(\mathrm{CH})$, hierba, BM).

Eryngium scaposum Turcz. (MAl-CET 1084, 1126 (CH), hierba, BM).

Micropleura renifolia Lag. (MAl-CET $1205(\mathrm{CH})$, hierba, BM/BME/BT/MA).

Sanicula liberta Cham. \& Schltdl. (MAI-CET $1053(\mathrm{CH})$, hierba, BM).

\section{Apocynaceae}

Asclepias similis HemsI. (MAI-CET 1098, 1306 (CH), hierba, BM/BME).

Gonolobus stenosepalus (Donn.Sm.) Woodson (MAI-CET $1073(\mathrm{CH})$, hierba, BM).

Metastelma schlechtendalii Decne. (MAI-CET $1185(\mathrm{CH})$, hierba, BM).

Vinca major L. (MAI-CET $1105(\mathrm{CH})$, hierba, PA).

\section{Aquifoliaceae}

Ilex sp. (MAl-CET $1248(\mathrm{CH})$, arbusto, BM).

\section{Araliaceae}

Hydrocotyle umbellata L. (Martínez-Icó \& Robles 647 (CH), hierba, BME/MA/PA).

Oreopanax arcanus A.C.Sm. (GOES $1583(\mathrm{CH})$, árbol, BM, leña, Mu'ktik nok'osik'al). (CR B2ab(iii,iv)**

Oreopanax xalapensis (Kunth) Decne. \& Planch. (MAI-CET $1199(\mathrm{CH})$, árbol, BM/BME/BT, forrajera y herramientas, Yich'akmut). (NT)**

\section{Asparagaceae}

Maianthemum flexuosum (Bertol.) LaFrankie (MAI-CET $1089(\mathrm{CH})$, hierba, BM/BME/BT).

Echeandia reflexa (Cav.) Rose (MAI-CET $1143(\mathrm{CH})$, hierba, CA/MA/PA).

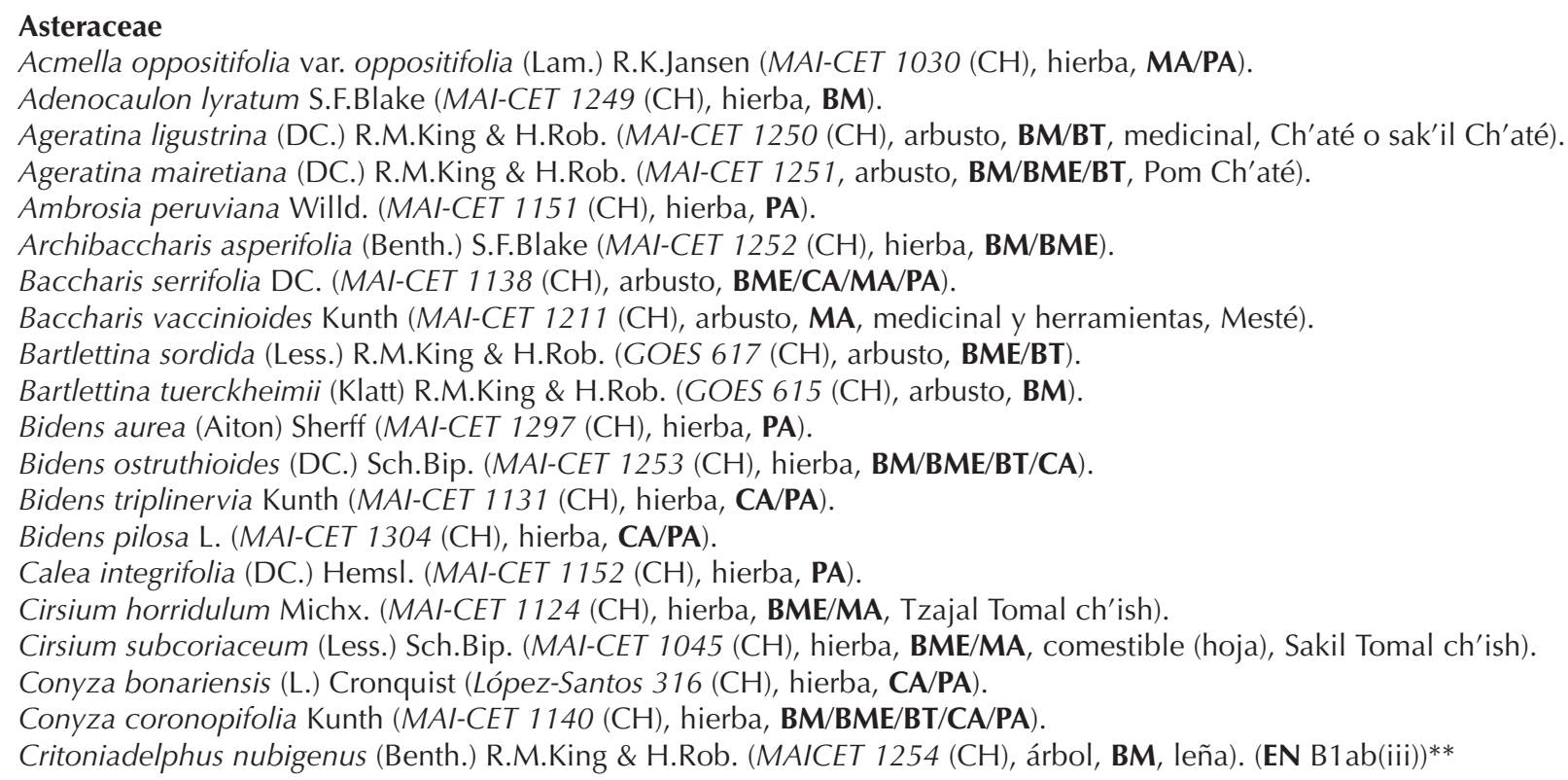


Apéndice 1. Continuación

Dahlia imperialis Roezl ex Ortgies (GOES $604(\mathrm{CH})$, hierba, CA/MA).

Dyssodia papposa (Vent.) Hitchc. (MAI-CET $1155(\mathrm{CH})$, hierba, CA).

Eupatorium pycnocephalum Less. (MAI-CET $1139(\mathrm{CH})$, arbusto, BM).

Galinsoga quadriradiata Cav. (MAI-CET $1255(\mathrm{CH})$, hierba, CA, comestible (hoja), Uskun).

Gnaphalium americanum Mill. (MAI-CET 1034 (CH), hierba, BM/BME/BT).

Hebeclinium macrophyllum (L.) DC. (MAI-CET $1177(\mathrm{CH})$, arbusto, BME/CA/MA).

Helenium scorzonerifolium (DC.) A.Gray (MAI-CET 1065 (CH), hierba, BM).

Hieracium abscissum Less. (MAI-CET $1111(\mathrm{CH})$, arbusto, BT/MA/PA).

Hymenostephium microcephalum (Less.) S.F.Blake (MAI-CET $1148(\mathrm{CH})$, arbusto, BM/BME/BT).

Jaegeria hirta (Lag.) Less. (MAI-CET 1256 (CH), hierba, CA/MA).

Melampodium montanum Benth. (MAI-CET $1257(\mathrm{CH})$, hierba, BM/BME).

Pinaropappus spathulatus Brandegee (MAI-CET $1128(\mathrm{CH})$, hierba, BT/CA).

Piqueria pilosa Kunth (MAI-CET $1258(\mathrm{CH})$, hierba, BM/BME/BT, forrajera, Pe'ch-chikin Womol).

Piqueria trinervia Cav. (López-Santos $304(\mathrm{CH})$, hierba, BM/BME/BT).

Pseudognaphalium chartaceum (Greenm.) Anderb. (GOES $594(\mathrm{CH})$, hierba, BM/BME/BT).

Roldana barba-johannis (DC.) H.Rob. \& Brettell (MAI-CET 1075 (CH), arbusto, BM/BME/BT, Yishperesh).

Roldana heterogama (Benth.) H.Rob. \& Brettell (MAI-CET $1195(\mathrm{CH})$, arbusto, BM/BME).

Roldana oaxacana (Hemsl.) H.Rob. \& Brettell (MAl-CET $1259(\mathrm{CH})$, arbusto, BM, medicinal, Sakil tutumishi'k).

Schistocarpha bicolor Less. (Ramírez-Marcial et al. $447(\mathrm{CH})$, arbusto, BM).

Senecio acutangulus (Bertol.) Hemsl. (MAI-CET $1260(\mathrm{CH})$, arbusto, BM, medicinal, ik'al tutumishi'k).

Senecio cobanensis J.M.Coult. (GOES $1827(\mathrm{CH})$, arbusto, BM).

Senecio doratophyllus Benth. (MAI-CET $1106(\mathrm{CH})$, hierba, BM/BME/BT, medicinal, Poshil i'k).

Senecio uspantanensis Greenm. (GOES $1828(\mathrm{CH})$, arbusto, BM/BME).

Sigesbeckia jorullensis Kunth (MAI-CET $1032(\mathrm{CH})$, hierba, BM, forrajera, Nap'ap Womol).

Simsia amplexicaulis (Cav.) Pers. (MAl-CET $1261(\mathrm{CH})$, hierba, BM/BME/BT).

Smallanthus oaxacanus (Sch.Bip. ex Klatt) H.Rob. (MAl-CET $1198(\mathrm{CH})$, hierba, MA/PA).

Sonchus asper (L.) Hill (MAl-CET $1090(\mathrm{CH})$, hierba, PA).

Stevia ovata Willd. (MAl-CET $1262(\mathrm{CH})$, hierba, CA).

Stevia serrata Cav. (MAl-CET $1263(\mathrm{CH})$, hierba, CA).

Symphyotrichum moranense (Kunth) G.L.Nesom (MAl-CET $1043(\mathrm{CH})$, hierba, PA).

Tagetes filifolia Lag. (MAI-CET $1264(\mathrm{CH})$, hierba, MA/PA).

Tagetes nelsonii Greenm. (MAl-CET $1298(\mathrm{CH})$, hierba, PA).

Tagetes lucida Cav. (MAI-CET $1300(\mathrm{CH})$, hierba, PA).

Taraxacum officinale F.H.Wigg. (MAI-CET 1074 (CH), hierba, PA, Diente de León).

Verbesina apleura S.F.Blake (GOES $990(\mathrm{CH})$, arbusto, BME/BT/MA).

Verbesina perymenioides Sch.Bip. ex Klatt (MAI-CET $1192(\mathrm{CH})$, arbusto, BM/BME/BT/MA, forrajera y leña, Such'alté). (LC)**

Vicia pulchella subsp. pulchella Kunth (GOES $681(\mathrm{CH})$, trepadora, CA/MA).

\section{Begoniaceae}

Begonia convallariodora C.DC. (GOES $2021(\mathrm{CH})$, hierba, BM).

Begonia oaxacana A.DC. (MAI-CET $1070(\mathrm{CH})$, hierba, BM, forrajera, Sikil Womol).

\section{Betulaceae}

Alnus acuminata Kunth (MAl-CET $1154(\mathrm{CH})$, árbol, BM, leña y herramientas, Nok). (LC)**

\section{Brassicaceae}

Lepidium oblongum Small (MAI-CET 1087 (CH), hierba, PA).

\section{Bromeliaceae}

Tillandsia caput-medusae E.Morren (CET-MAI $218(\mathrm{CH})$, hierba, BM).

Tillandsia eizii L.B.Sm. (CET-MAI 217 (CH), hierba, BM).

Tillandsia guatemalensis L.B.Sm. (CET-MAI $190(\mathrm{CH})$, hierba, BM).

Tillandsia lautneri Ehlers. (CET-MAI $215(\mathrm{CH})$, hierba, BM).

Tillandsia ponderosa L.B.Sm. (CET-MAI $202(\mathrm{CH})$, hierba, BM). (A)*

Tillandsia vicentina Standl. (CET-MAI $216(\mathrm{CH})$, hierba, BM). 
Apéndice 1. Continuación

Calceolariaceae

Calceolaria mexicana Benth. (MAI-CET $1302(\mathrm{CH})$, hierba, PA).

\section{Campanulaceae}

Centropogon grandidentatus (Schltdl.) Zahlbr. (MAI-CET $1060(\mathrm{CH})$, hierba, BM, Mutka Ichil Womol). Lobelia nana Kunth (MAI-CET $1061(\mathrm{CH})$, hierba, PA).

Lobelia sartorii Vatke (MAl-CET $1102(\mathrm{CH})$, hierba, BM/BME/BT).

\section{Caryophyllaceae}

Arenaria guatemalensis Standl. \& Steyerm. (MAI-CET $1168(\mathrm{CH})$, hierba, BM/BME).

Drymaria hypericifolia Briq. (MAI-CET $1246(\mathrm{CH})$, hierba, BM).

\section{Celastraceae}

Celastrus vulcanicola Donn.Sm. (GOES $618(\mathrm{CH})$, trepadora, BM, mecate, Vach a'k).

\section{Chloranthaceae}

Hedyosmum mexicanum C.Cordem. (Martínez-lcó et al. $31(\mathrm{CH})$, árbol, BM). (LC)**

\section{Cistaceae}

Helianthemum glomeratum (Lag.) Lag. ex Dunal (MAI-CET 1133 (CH), hierba, CA/PA).

\section{Clethraceae}

Clethra occidentalis (L.) Kuntze (MAl-CET $1212(\mathrm{CH})$, árbol, BME/BT, leña, Asté).

\section{Commelinaceae}

Commelina diffusa Burm.f. (MAI-CET $1156(\mathrm{CH})$, hierba, BM, Tz'emeni).

Commelina erecta L. (MAI-CET $1220(\mathrm{CH})$, hierba, BM, Tz'emeni).

Tinantia erecta (Jacq.) Schltdl. (López-Santos et al. 312, MAl-CET 1299 (CH), hierba, BME/CA).

Tradescantia commelinoides Schult. \& Schult.f. (MAI-CET $1116(\mathrm{CH})$, hierba, BM/BME/BT, Tz'emeni).

\section{Convolvulaceae}

Dichondra sericea Sw. (MAl-CET $1265(\mathrm{CH})$, hierba, BME/CA/MA/PA).

Ipomoea purpurea (L.) Roth (MAI-CET $1141(\mathrm{CH})$, hierba, CA/MA/PA).

Ipomoea orizabensis (G.Pelletan) Ledeb. ex Steud. (MAI-CET 1305 (CH), hierba, CA/MA/PA).

\section{Coriariaceae}

Coriaria ruscifolia L. (MAI-CET $1104(\mathrm{CH})$, arbusto, CA/MA).

\section{Cornaceae}

Cornus disciflora DC. (MAl-CET $1213(\mathrm{CH})$, árbol, BM, leña, Sakilté). (VU B1ab(iii))**

Cornus excelsa Kunth (MAI-CET $1145(\mathrm{CH})$, árbol, BM/BME/BT, leña y herramientas, Isbon).

\section{Cucurbitaceae}

Cyclanthera pedata (L.) Schrad. (MAI-CET $1233(\mathrm{CH})$, trepadora, BT, jabón, Polotz).

Cyclanthera langaei Cogn. (GOES 2023 (CH), hierba, PA).

\section{Cyperaceae}

Cyperus odoratus L. (MAI-CET 1044, $1142(\mathrm{CH})$, hierba, PA).

Uncinia hamata (Sw.) Urb. (GOES 603 (CH), hierba, BM/BME/BT/PA).

\section{Ericaceae}

Arbutus xalapensis Kunth (MAI-CET $1157(\mathrm{CH})$, árbol, BM/BME, carbón, leña y postes, On-te). (LC)** Chimaphila maculata (L.) Pursh (MAI-CET 1266 (CH), hierba, BME).

Chimaphila umbellata (L.) W.P.C.Barton (MAI-CET $1267(\mathrm{CH})$, hierba, BM).

Gaultheria acuminata Schltdl. \& Cham. (MAI-CET $1268(\mathrm{CH})$, arbusto, BM).

Vaccinium confertum Kunth (Ramírez-Marcial $664(\mathrm{CH})$, arbusto, BT/CA/MA/PA). 
Apéndice 1. Continuación

\section{Euphorbiaceae}

Acalypha arvensis Poepp. (MAI-CET 1310 (CH), hierba, CA/PA).

\section{Fabaceae}

Acacia angustissima (Mill.) Kuntze (MAI-CET $1166(\mathrm{CH})$, arbusto, BT, Shashim mut, Pukuj).

Cologania broussonetii (Balb.) DC. (MAI-CET $1234(\mathrm{CH})$, trepadora, BME/BT).

Crotalaria pumila Ortega (MAI-CET $1278(\mathrm{CH})$, hierba, CA/PA).

Dalea leporina (Aiton) Bullock (GOES 593, MAl-CET 1301 (CH), hierba, CA/PA).

Desmodium aparines (Link) DC. (MAI-CET $1160(\mathrm{CH})$, hierba, BME/CA/MA).

Desmodium palmeri Hemsl. (MAl-CET $1132(\mathrm{CH})$, hierba, BT/CA/MA/PA).

Lupinus aschenbornii S.Schauer (MAI-CET $1025(\mathrm{CH})$, hierba, CA/PA).

Medicago polymorpha L. (MAI-CET $1136(\mathrm{CH})$, hierba, CA/MA/PA).

Trifolium amabile Kunth (MAl-CET $1171(\mathrm{CH})$, hierba, CA/MA/PA).

Trifolium repens L. (MAI-CET $1038(\mathrm{CH})$, hierba, PA).

\section{Fagaceae}

Quercus benthamii A.DC. (MAl-CET 1206 (CH), árbol, BM, leña, carbón y postes). (EN A3c; B1ab(ii,iii,iv,v))**

Quercus crassifolia Bonpl. (MAl-CET 1097, $1196(\mathrm{CH})$, árbol, BM/BME/BT/MA, leña, carbón y postes, Bochje o Batzt'zé). (LC)**

Quercus crispipilis Trel. (GOES $646(\mathrm{CH})$, árbol, BM, leña, carbón y postes). (VU A4c; B1 ab(ii,iii)**

Quercus laurina Bonpl. (MAl-CET $1163(\mathrm{CH})$, árbol, BM/BME/BT/MA, leña, carbón y postes, Chiquinib). (LC)**

Quercus rugosa Née (GOES $216(\mathrm{CH})$, árbol, BME/BT, leña, carbón, construcción, K'antulante). (LC)**

\section{Garryaceae}

Garrya laurifolia Hartw. ex Benth. (MAI-CET $1147(\mathrm{CH})$, árbol, BM, medicinal y leña, Ik'al vini'kte). (VU A4c; B2ab(iii))**

\section{Gereniaceae}

Geranium repens H.E.Moore (Martínez-Icó \& Robles $650(\mathrm{CH})$, hierba, BM/BME).

Geranium seemannii Peyr. (MAl-CET 1129, 1201 (CH), hierba, BM/BME).

Geranium vulcanicola Small (MAl-CET $1083(\mathrm{CH})$, hierba, BM/BME).

\section{Hypericaceae}

Hypericum hypericoides (L.) Crantz (MAI-CET 1041 (CH), hierba, PA).

\section{Hypoxidaceae}

Hypoxis decumbens L. (MAI-CET $1044(\mathrm{CH})$, hierba, PA).

\section{Iridaceae}

Crocosmia $\times$ crocosmiiflora (Lemoine) N.E.Br. (MAl-CET $1114(\mathrm{CH})$, hierba, CA/PA).

Orthrosanthus chimboracensis (Kunth) Baker (MAl-CET $1112(\mathrm{CH})$, hierba, BME/BT/MA, Shanil Womol).

Sisyrinchium convolutum Nocca (MAl-CET $1117(\mathrm{CH})$, hierba, BT).

Tigridia chiapensis Molseed ex Cruden (MAl-CET $1056(\mathrm{CH})$, hierba, PA).

Tigridia pavonia (L.f.) DC. (MAl-CET 1086 (CH), hierba, PA).

\section{Juncaceae}

Juncus effusus L. (MAI-CET $1109(\mathrm{CH})$, hierba, PA).

\section{Lamiaceae}

Clinopodium brownei (Sw.) Kuntze (MAI-CET 1069 (CH), hierba, PA, medicinal (hoja), Mujil Jomol). Lepechinia schiedeana (Schltdl.) Vatke (MAI-CET $1100(\mathrm{CH})$, hierba, BME/BT/CA/MA/PA).

Prunella vulgaris L. (MAl-CET $1033(\mathrm{CH})$, hierba, BM/BME/BT/PA).

Salvia cinnabarina M.Martens \& Galeotti (MAl-CET $1054(\mathrm{CH})$, hierba, BM).

Salvia karwinskii Benth (MAl-CET 1095 (CH), arbusto, BME/BT, Muktik omtz'unun).

Salvia lavanduloides Kunth (MAl-CET $1165(\mathrm{CH})$, hierba, BME/BT/CA/MA).

Salvia patens Cav. (Chame \& Luna $214(\mathrm{CH})$, hierba, CA/MA/PA).

Stachys agraria Schltdl. \& Cham (MAI-CET $1175(\mathrm{CH})$, hierba, CA/PA).

Stachys coccinea Ortega (MAI-CET $1194(\mathrm{CH})$, hierba, CA/PA). 
Apéndice 1. Continuación

\section{Lauraceae}

Cassytha filiformis L. (MAI-CET $1118(\mathrm{CH})$, trepadora, BM/CA/MA).

Litsea glauscesens Kunth (MAl-CET $1188(\mathrm{CH})$, árbol, BM/BME/BT, leña y condimento, Tzij uch, laurel). (VU A2)**

Ocotea acuminatissima (Lundell) Rohwer (MAI-CET 1217 (CH), arbusto, BM). (VU B1ab(iii))**

Persea americana Mill. (GOES 609 (CH), árbol, BM, comestible (fruto), Tz'itz-mut). (EN B1ab(iii))**

Persea donnell-smithii Mez (Luna-Gómez 527 (CH), árbol, BM). (VU B1ab(iii)**

\section{Lythraceae}

Cuphea aequipetala Cav. (MAI-CET $1093(\mathrm{CH})$, hierba, CA).

Cuphea hyssopifolia Kunth (MAI-CET $1173(\mathrm{CH})$, hierba, CA/MA/PA).

\section{Magnoliaceae}

Magnolia sharpii Miranda (Chamé \& Luna $217(\mathrm{CH})$, árbol, BM, leña y las hojas para tamales, tojchó). (EN B1ab(i,iii).C2a(i))**

\section{Malvaceae}

Sida acuta Burm.f. (MAI-CET $1178(\mathrm{CH})$, hierba, CA).

\section{Melastomataceae}

Miconia glaberrima (Schltdl.) Naudin (GOES 2025 (CH), árbol, BM).

Miconia hemenostigma Naudin (MAI-CET $1235(\mathrm{CH})$, arbusto, BM). (EN A4c; B1ab(iii))**

Miconia oligotricha (DC.) Naudin (MAl-CET 1207 (CH), arbusto, BM, leña, Chi'ilte). (NT)**

\section{Myricaceae}

Myrica cerifera L. (MAl-CET $1162(\mathrm{CH})$, árbol, BM/BME, leña, Satín). (LC)**

\section{Onagraceae}

Fuchsia encliandra Steud. (MAI-CET $1200(\mathrm{CH})$, arbusto, BM).

Fuchsia microphylla Kunth (MAI-CET 1047 (CH), arbusto, BM, Vik'tal Alperez Womol).

Fuchsia paniculata Lindl. (MAl-CET $1051(\mathrm{CH})$, árbol, BM, ornato y leña, Atz'am nichim).

Fuchsia splendens Zucc. (MAI-CET $1066(\mathrm{CH})$, arbusto, BM, Muktik Alperez Womol).

Fuchsia thymifolia Kunth (MAI-CET 1037 (CH), arbusto, BM/BME/BT).

Lopezia racemosa Cav. (MAl-CET $1303(\mathrm{CH})$, hierba, BME/CA/MA/PA).

Oenothera pubescens Willd. ex Spreng. (MAI-CET 1039 (CH), hierba, CA/PA).

Oenothera rosea L'Hér.ex Aiton (MAI-CET $1040(\mathrm{CH})$, hierba, CA/PA).

\section{Orchidaceae}

Calanthe calanthoides (A.Rich. \& Galeotti) Hamer \& Garay (CET-MAI 191 (CH, CICY), hierba, terrestre, BM). Corallorhiza bulbosa A.Rich. \& Galeotti (CET et al. 222 (CH, CICY), hierba, terrestre, BM). Epidendrum eximium L.O.Williams (Castillo-Hernández $2368(\mathrm{CH})$, hierba, epífita, BM). Epidendrum microcharis Rchb.f. (Wolf $1658(\mathrm{CH})$, hierba, epífita, BM).

Govenia superba (LaLlave \& Lex.) Lindl. (Chamé \& Luna $220(\mathrm{CH})$, hierba, terrestre, BM). Liparis arnoglossophylla (Rchb.f.) Rchb.f. (CET-MAI $192 \mathrm{CH}, \mathrm{CICY}$ ), hierba, terrestre, BM/BME). Malaxis brachyrrhynchos (Rchb.f.) Ames (CET-MAI 193, 211 (CH, CICY), hierba, terrestre, BM). Ponthieva schaffneri (Rchb.f.) E.W.Greenw. (GOES $1576(\mathrm{CH})$, hierba, terrestre, BM) Prescottia stachyodes (Sw.) Lindl. (CET et al. 221 (CH, CICY), hierba, terrestre, BM). Prosthechea varicosa (Bateman ex Lindl.) W.E.Higgins (Wolf $2417(\mathrm{CH})$, hierba, epífita, BM). Prosthechea vitellina (Lindl.) W.E.Higgins (CET-MAl 189, 212 (CH, CICY), hierba, epífita, BM). (Pr)* Rhynchostele bictoniensis (Bateman) Soto Arenas \& Salazar (Chamé \& Luna $218(\mathrm{CH})$, CET-MAI $248(\mathrm{CH}, \mathrm{CICY})$ hierba, terrestre, BM). Rhynchostele stellata (Lindl.) Soto Arenas \& Salazar (Wolf 2428 (CH), CET et al. 283 (CICY), hierba, epífita, BM).

\section{Orobanchaceae}

Castilleja tapeinoclada Loes. (MAI-CET $1055(\mathrm{CH})$, hierba, CA/MA/PA).

\section{Oxalidaceae}

Oxalis alpina (Rose) Rose ex R.Knuth (MAI-CET 1057 (CH), hierba, BME/CA/MA/PA).

Oxalis corniculata L. (MAI-CET $1036(\mathrm{CH})$, hierba, BME/CA/MA/PA).

Oxalis divergens Benth. ex Lindl. (MAI-CET $1181(\mathrm{CH})$, hierba, BM, Pojol). 
Apéndice 1. Continuación

\section{Papaveraceae}

Bocconia gracilis Hutch. (MAI-CET $1231(\mathrm{CH})$, arbusto, BM). (EN B2ab(iii))**

Passifloraceae

Passiflora membranacea Benth. (MAI-CET $1052(\mathrm{CH})$, trepadora, BM, mecate, Canario).

\section{Pentaphylacaceae}

Cleyera theoides (Sw.) Choisy (MAl-CET $1187(\mathrm{CH})$, árbol, BM/BME/BT, leña y construcción, Coshoshté). (NT)**

Ternstroemia lineata DC. (MAI-CET $1184(\mathrm{CH})$, árbol, BM, leña, Bix'tal kaxlan tilil). (NT)**

\section{Phrymaceae}

Mimulus glabratus Kunth (Martínez-Icó $680(\mathrm{CH})$, hierba, BM).

\section{Phytolaccaceae}

Phytolacca icosandra L. (MAl-CET 1096 (CH), hierba, BME, comestible (hoja), J'om).

\section{Plantaginaceae}

Plantago australis Lam. (MAl-CET 1058 (CH), hierba, BT/CA/MA/PA).

Plantago major L. (MAl-CET 1059 (CH), hierba, BT/CA/MA/PA).

\section{Piperaceae}

Peperomia deppeana Schltdl. \& Cham. (MAI-CET $1218(\mathrm{CH})$, hierba, BM).

Peperomiagalioides Kunth (MAI-CET $1082(\mathrm{CH})$, hierba, BM).

Peperomia quadrifolia (L.) Kunth (MAI-CET $1219(\mathrm{CH})$, hierba, BM).

Peperomia rotundifolia (L.) Kunth (MAI-CET $1081(\mathrm{CH})$, hierba, BM).

\section{Poaceae}

Aegopogon cenchroides Humb. \& Bonpl. ex Willd. (MAl-CET $1221(\mathrm{CH})$, hierba, CA/MA/PA).

Axonopus fissifolius (Raddi) Kuhlm (MAl-CET $1222(\mathrm{CH})$, hierba, PA).

Brachypodium mexicanum (Roem. \& Schult.) Link (MAl-CET $1223(\mathrm{CH})$, hierba, BM/BME).

Bromus carinatus Hook. \& Arn. (MAI-CET $1119(\mathrm{CH})$, hierba, CA, forrajera (para conejos), K'ut'ul).

Eragrostis mexicana (Hornem.) Link (MAI-CET $1120(\mathrm{CH})$, hierba, CA/MA/PA).

IChnanthus tenuis (J.Presl \& C.Presl) Hitchc. \& Chase (MAI-CET 1121, 1122 (CH), hierba, BME/BT/CA/MA).

Muhlenbergia vaginata Swallen (MAI-CET $1224(\mathrm{CH})$, hierba, PA, construcción de techos, Jovel).

Panicum laxiflorum Lam. (MAl-CET $1082(\mathrm{CH})$, hierba, BME/CA/PA).

Paspalum jaliscanum Chase (MAI-CET $1225(\mathrm{CH})$, hierba, CA/MA/PA).

Paspalum minus E.Fourn. (MAI-CET $1226(\mathrm{CH})$, hierba, CA/MA/PA).

Pennisetum clandestinum Hochst. ex Chiov. (MAI-CET $1150(\mathrm{CH})$, hierba, CA/PA).

Poa annua L. (MAI-CET 1146 (CH), hierba, CA/PA).

Setaria parviflora (Poir.) Kerguélen (MAl-CET $1158(\mathrm{CH})$, hierba, CA/MA/PA).

Sporobolus indicus (L.) R.Br. (MAI-CET $1135(\mathrm{CH})$, hierba, CA/PA, forrajera, Vikt'al).

Triniochloa stipoides (Kunth) Hitchc. (MAI-CET 1227 (CH), hierba, BME/BT/MA).

Vulpia bromoides (L.) Gray (MAl-CET $1228(\mathrm{CH})$, hierba, CA/MA/PA).

Zeugites americanus Willd. (MAI-CET $1107(\mathrm{CH})$, hierba, BM/BME/BT/MA).

\section{Polygalaceae}

Monnina xalapensis Kunth (MAI-CET $1048(\mathrm{CH})$, arbusto, BM/BME/BT/MA, medicinal, Pitz'otz).

\section{Polygonaceae}

Polygonum punctatum Buch.-Ham. ex D.Don (MAl-CET $1174(\mathrm{CH})$, hierba, CA/PA).

Rumex obtusifolius L. (MAI-CET $1042(\mathrm{CH})$, hierba, PA).

\section{Primulaceae}

Anagallis arvensis L. (MAI-CET $1088(\mathrm{CH})$, hierba, PA).

Myrsine juergensenii (Mez) Ricketson \& Pipoly (MAI-CET 1067 (CH), árbol, BM/BME/BT, leña, herramientas y rituales religiosos, Tilil). (NT)**

Parathesis chiapensis Fernald (GOES $577(\mathrm{CH})$, arbusto, BM). (VU B1ab(iii) $)^{* *}$ 
Apéndice 1. Continuación

Parathesis donnell-smithii Mez (GOES $2031(\mathrm{CH})$, arbusto, BM). (NT)**

Parathesis leptopa Lundell (MAl-CET $1113(\mathrm{CH})$, arbusto, BM, Tililja o Bak'e Lamóch). (EN B1ab(iii))**

Parathesis sessilifolia Donn.Sm. (GOES 569 (CH), arbusto, BM).

\section{Ranunculaceae}

Clematis dioica L. (MAI-CET $1167(\mathrm{CH})$, trepadora, BM).

Ranunculus petiolaris Humb., Bonpl. \& Kunth ex DC. (MAl-CET 1049 (CH), hierba, BM/BME/BT/PA, medicinal (anestésico), Mitank'ak)

\section{Rhamnaceae}

Ceanothus caeruleus Lag. (MAI-CET $1180(\mathrm{CH})$, arbusto, BME/BT/MA).

Frangula mucronata (Schltdl.) Grubov (MAl-CET $1236(\mathrm{CH})$, arbusto, BM/BME). (LC)**

Rhamnus mcvaughii L.A.Johnst. \& M.C.Johnst. (GOES $584(\mathrm{CH})$, arbusto, BM/BME).

Rhamnus sharpii M.C.Johnst. \& L.A.Johnst. (MAI-CET 1209 (CH), árbol, BM, leña, K'anté). (LC)**

\section{Rosaceae}

Agrimonia macrocarpa (Focke) Rydb. (MAI-CET 1237 (CH), hierba, BME).

Alchemilla pringlei (Rydb.) Fedde (MAl-CET $1238(\mathrm{CH})$, hierba, BM, Tz'jul Womol).

Crataegus pubescens (C.Presl) C.Presl (MAI-CET $1149(\mathrm{CH})$, árbol, BM/BT, Ch'ish, Ch'ishté).

Fragaria vesca L. (MAI-CET 1035 (CH), hierba, BM/BME/BT/CA/PA).

Holodiscus argenteus (L.f.) Maxim. (GOES 991 (CH), arbusto, BM, leña y herramientas, Pomosté, Tzajalté). (LC)**

Lachemilla aphanoides (Mutis ex L.f.) Rothm. (MAl-CET $1202(\mathrm{CH})$, hierba, CA/MA/PA, Tz'jul Womol).

Lachemilla orbiculata (Ruiz \& Pav.) Rydb. (López-Santos $324(\mathrm{CH})$, hierba, PA).

Lachemilla pectinata (Kunth) Rothm. (MAI-CET 1068 (CH), hierba, PA).

Photinia microcarpa Standl. (Martínez-Icó $626(\mathrm{CH})$, árbol, BM). (VU A4c)**

Prunus rhamnoides Koehne (MAl-CET $1210(\mathrm{CH})$, árbol, BM, postes y leña, Tz'utujté). (VU A1c; B1ab(iii))**

Prunus serotina Ehrh. (MAI-CET $1239(\mathrm{CH})$, árbol, BM/BME/BT, postes y leña, Ch'ishté). (LC)**

Rubus adenotrichos Schltdl. (MAI-CET $1190(\mathrm{CH})$, arbusto, CA/MA/PA).

Rubus coriifolius Liebm. (MAI-CET $1240(\mathrm{CH})$, arbusto, BM, comestible (hoja), Buluk-citmakun, Pilizat).

Rubus sapidus Schltdl. (MAl-CET 1203 (CH), arbusto, BM).

\section{Rubiaceae}

Arcytophyllum serpyllaceum (Schltdl.) Terrell (MAI-CET 1080 (CH), hierba, CA/MA/PA).

Crusea calocephala DC. (MAI-CET 1269 (CH), hierba, BM/BME/BT/CA/PA).

Galium aschenbornii Nees \& S.Schauer (MAI-CET $1270(\mathrm{CH})$, hierba, BME/BT).

Galium mexicanum Kunth (MAI-CET $1115(\mathrm{CH})$, hierba, CA/MA).

Nertera granadensis (Mutis ex L.f.) Druce (MAl-CET $1091(\mathrm{CH})$, hierba, BM/BME/BT).

Psychotria costivenia var. costivenia Griseb (GOES $596(\mathrm{CH})$, arbusto, BM).

Psychotria minarum Standl. \& Steyerm. (GOES $1575(\mathrm{CH})$, arbusto, BT).

Rogiera amoena Planch. (GOES $654(\mathrm{CH})$, árbol, BM/BME). (VU B1ab(iii)**

Spermacoce laevis Lam. (MAI-CET $1130(\mathrm{CH})$, hierba, BT/CA/MA/PA).

\section{Rutaceae}

Zanthoxylum foliolosum Donn.Sm. (MAI-CET $1182(\mathrm{CH})$, trepadora, BM/BME, Leben'tzotz).

Zanthoxylum melanostictum Schltdl. \& Cham. (MAI-CET $1242(\mathrm{CH})$, arbusto, BM, leña). (LC)**

\section{Salicaceae}

Olmediella betschleriana (Göpp.) Loes. (Chamé \& Luna $215(\mathrm{CH})$, árbol, BM, leña, K'olomash). (EN A4c; B1ab(iii))** Xylosma quichensis Donn.Sm. (MAI-CET $1243(\mathrm{CH})$, arbusto, BM, Ch'ish bolom).

\section{Santalaceae}

Phoradendron tonduzii Trel. (MAI-CET $1294(\mathrm{CH})$, hierba, parasita, BM).

\section{Scrophulariaceae}

Buddleja cordata Kunth (MAI-CET $1271(\mathrm{CH})$, árbol, BT, forrajera, leña y postes, Tzelepat). (EN A4c; B1ab(iii))**

Buddleja crotonoides A.Gray (MAI-CET $1164(\mathrm{CH})$, arbusto, BT/CA/MA/PA, Tzelepat). 
Apéndice 1. Continuación

\section{Smilacaceae}

Smilax domingensis Willd. (Ramírez-Marcial $670(\mathrm{CH})$, trepadora, BM).

Smilax jalapensis Schltdl. (MAl-CET $1208(\mathrm{CH})$, trepadora, BM, mecate, A'k).

Smilax laurifolia L. (Ramírez-Marcial $450(\mathrm{CH})$, trepadora, BM, artesanías, Kulch'ix, Kul a'k).

Smilax mollis Humb. \& Bonpl. ex Willd. (MAI-CET 1229 (CH), trepadora, BM/BME/BT).

Smilax spinosa Mill. (MAl-CET $1230(\mathrm{CH})$, trepadora, BM, $\left.\mathrm{A}^{\prime} \mathrm{k}\right)$.

\section{Sapindaceae}

Acer negundo subsp. mexicanum (DC.) Wesm. (MAI-CET $1063(\mathrm{CH})$, árbol, PA). (VU B1ab(ii,ii,iv,v))**

\section{Solanaceae}

Cestrum aurantiacum Lindl. (MAI-CET 1204 (CH), arbusto, BM/BME). (LC)**

Cestrum guatemalense Francey (MAI-CET $1189(\mathrm{CH})$, arbusto, BM/BME/BT).

Lycianthes chiapensis (Brandegee) Standl. (GOES 2026 (CH), hierba, MA/PA).

Lycianthes connata J.L.Gentry (MAI-CET $1272(\mathrm{CH})$, arbusto, BM).

Lycianthes gorgonea Bitter (Ramírez-Marcial $631(\mathrm{CH})$, hierba, CA).

Lycianthes purpusii (Brandegee) Bitter (MAI-CET 1031 (CH), trepadora, BM, Chilol antivo).

Physalis gracilis Miers (MAI-CET $1099(\mathrm{CH})$, hierba, BM/BT/CA, Yashal tuilté, Tuilté).

Solanum aligerum Schltdl. (MAl-CET $1108(\mathrm{CH})$, arbusto, BM/BME).

Solanum americanum Mill. (MAI-CET $1094(\mathrm{CH})$, hierba, BM, comestible (hoja), Yerbamora).

Solanum aphyodendron S.Knapp (MAI-CET $1092(\mathrm{CH})$, arbusto, BM/BME).

Solanum appendiculatum Dunal (MAl-CET $1186(\mathrm{CH})$, hierba, BM, Chichol A'k,).

Solanum lanceolatum Cav. (MAI-CET $1110(\mathrm{CH})$, arbusto, BME/MA).

Solanum nigricans M.Martens \& Galeotti (MAI-CET $1046(\mathrm{CH})$, arbusto, BM/BME/BT, Yashal tuilté, Tuilté). (LC)**

Solanum rudepannum Dunal (GOES $578(\mathrm{CH})$, arbusto, BME/BT/MA).

\section{Styracaceae}

Styrax magnus Lundell (MAI-CET $1273(\mathrm{CH})$, árbol, BM). (LC)**

\section{Symplocaceae}

Symplocos limoncillo Bonpl. (GOES 430 (CH), árbol, BM/BME/BT, leña, Yashal-té). (VU A4c; B1ab(iii))**

\section{Thymelaeaceae}

Daphnopsis selerorum Gilg (MAl-CET 1244 (CH), arbusto, BM).

\section{Winteraceae}

Drimys granadensis L.f. (GOES $576(\mathrm{CH})$, árbol, BM). (VU A4c; B1ab(iii))**

\section{Verbenaceae}

Citharexylum donnell-smithii Greenm. (MAICET $1274(\mathrm{CH})$, árbol, BM).

Lantana camara L. (MAI-CET $1064(\mathrm{CH})$, arbusto, PA).

Lantana hispida Kunth (MAl-CET 1123, $1137(\mathrm{CH})$, arbusto, CA/MA/PA).

Verbena carolina L. (MAl-CET $1275(\mathrm{CH})$, arbusto, PA).

\section{Violaceae}

Viola hookeriana Kunth (MAl-CET $1134(\mathrm{CH})$, hierba, BT/CA/MA/PA).

Viola nannei Pol. (MAI-CET $1062(\mathrm{CH})$, hierba, PA).

Viola sacchalinensis var. sacchalinensis H.Boissieu (MAI-CET $1245(\mathrm{CH})$, hierba, CA/MA/PA).

\section{Vitaceae}

Cissus trianae Planch. (MAI-CET $1295(\mathrm{CH})$, trepadora, BM). 